\title{
Weak and strong convergence theorems of a multistep iteration to a common fixed point of a family of nonself asymptotically nonexpansive mappings in banach spaces
}

\author{
Shrabani BanerJee and Binayak S.Choudhury \\ Department of Mathematics, \\ Bengal Engineering and Science University, \\ Shibpur, Howrah-711103, India. \\ email: banerjee.shrabani@yahoo.com, binayak12@yahoo.co.in
}

\begin{abstract}
In this paper we have defined a multistep iterative scheme with errors involving a family of asymptotically nonexpansive nonself mappings in Banach spaces. A retraction has been used in the construction of the iteration. We prove here weak and strong convergences of the iteration to common fixed points of the family of asymptotically nonexpansive nonself mappings. We have used several concepts of Banach space geometry. Our results improve and extend some recent results.
\end{abstract}

\section{RESUMEN}

En este artículo definimos un esquema de multi paso iterativo con errores que involucran una familia de aplicaciones no expansivas y no auto asintóticamente en espacios de Banach. Una retracción se ha usado en la construcción de la iteración. Probamos convergencias débiles y fuertes de las iteraciones a puntos fijos clásicos de la familia de aplicaciones no expansivas no auto asintóticamente. Hemos usado varios conceptos de geometría en espacios de Banach. Nuestro resultado mejora y extiende algunos resultados recientes.

Keywords and Phrases: Modified multistep iterative process with errors; nonself asymptotically nonexpansive mapping; retraction; Opial's condition; uniformly convex Banach space; common fixed point; Kadec-klee property; Condition $(\overline{\mathrm{B}})$; weak and strong convergence.

2010 AMS Mathematics Subject Classification: 47H10 


\section{Introduction}

Let $\mathrm{K}$ be a nonempty subset of real normed space $\mathrm{E}$. A self mapping $\mathrm{T}: \mathrm{K} \rightarrow \mathrm{K}$ is called nonexpansive if

$$
\|T x-T y\| \leq\|x-y\|, \text { for all } x, y \in K
$$

A self mapping $T: K \rightarrow K$ is called asymptotically nonexpansive if there exists a sequence $\left\{k_{n}\right\} \subset$ $[1, \infty)$ with $\lim _{n \rightarrow \infty} k_{n}=1$ such that

$$
\left\|T^{n} x-T^{n} y\right\| \leq k_{n}\|x-y\| \text { for all } x, y \in K \text { and } n \geq 1 \text {. }
$$

$\mathrm{T}$ is said to be uniformly L-Lipschitzian if there exists a constant $\mathrm{L}>0$ such that

$$
\left\|T^{n} x-T^{n} y\right\| \leq L\|x-y\| \text { for all } x, y \in K \text { and } n \geq 1 .
$$

The class of asymptotically nonexpansive mappings was introduced by Goebel and Kirk [7] in 1972 as a generalization of the class of nonexpansive self mappings. They proved that if $\mathrm{T}$ is a selfmap on $\mathrm{K}$ where $\mathrm{K}$ is a nonempty closed convex subset of a real uniformly convex Banach space, then $\mathrm{T}$ has a fixed point. Fixed point iterative processes for asymptotically nonexpansive self-mappings on convex subsets of Banach spaces have been studied extensively by many authors. Since T remains a self- mapping of a nonempty closed convex subset $\mathrm{K}$ of a Banach space $\mathrm{E}$, the well known Mann[1] and Ishikawa 8 iterative processes are well defined. If however the domain $\mathrm{K}$ of $\mathrm{T}$ is a proper subset of $E$ (and it is the case of several applications) and $T$ maps $K$ into $E$, then the iteration processes of Mann and Ishikawa and their modifications fail to be well defined. To overcome this problem Chidume et al. 2] introduced the concept of nonself asymptotically nonexpansive mappings in 2003 as a generalization of asymptotically nonexpansive self mappings. A subset $\mathrm{K}$ of $\mathrm{E}$ is said to be a retract of $E$ if there exists a continuous mapping $P: E \rightarrow K$ such that $P x=x$ for all $x \in K$. Every closed convex subset of a uniformly convex Banach space is a retract. A mapping $P: E \rightarrow E$ is said to be a retraction if $\mathrm{P}^{2}=\mathrm{P}$. It follows that if a map $\mathrm{P}$ is a retraction then $\mathrm{Py}=\mathrm{y}$ for all $\mathrm{y}$ in the range of $P$. The nonself asymptotically nonexpansive mapping is defined as follows:

Definition 1.1. (2 ) Let $\mathrm{E}$ be a real normed linear space, $\mathrm{K}$ be a nonempty subset of $\mathrm{E}$ and $\mathrm{P}: \mathrm{E} \rightarrow \mathrm{K}$ be the nonexpansive retraction of $E$ onto $K$. Let $T: K \rightarrow E$ be a non-self mapping. $T$ is said to be a non-self asymptotically nonexpansive mapping if there exists a sequence $\left\{k_{n}\right\} \subset[1, \infty)$ with $k_{n} \rightarrow 1$ as $n \rightarrow \infty$ such that the following inequality holds:

$$
\left\|T(P T)^{n-1} x-T(P T)^{n-1} y\right\| \leq k_{n}\|x-y\| \text {, for all } x, y \in K \text { and } n \geq 1 .
$$

$\mathrm{T}$ is said to be uniformly L-Lipschitzian if there exists a constant $\mathrm{L}>0$ such that

$$
\left\|T(P T)^{n-1} x-T(P T)^{n-1} y\right\| \leq L\|x-y\| \text {, for all } x, y \in K \text { and } n \geq 1 .
$$

If $T$ is a self map, then $P$ becomes the identity map so that (1.3) and (1.4) coinside with (1.1) and (1.2) respectively. 
Chidume et al. 2] introduced and studied the weak and strong convergences of the following iterative process

$$
\left\{\begin{array}{l}
x_{1} \in K \\
x_{n+1}=P\left(\left(1-\alpha_{n}\right) x_{n}+\alpha_{n} T(P T)^{n-1} x_{n}\right)
\end{array}\right.
$$

where $\left\{\alpha_{n}\right\}$ is a appropriate real sequence in $[0,1]$. If $T$ is a self map, then $P$ becomes the identity map so that (1.5) reduces to the Mann-type iteration scheme[11. Then Wang [19] used a similar scheme to prove the weak and strong convergence theorems for a pair of non-self asymptotically nonexpansive mappings which is given by

$$
\left\{\begin{array}{l}
x_{1} \in K \\
x_{n+1}=P\left(\left(1-\alpha_{n}\right) x_{n}+\alpha_{n} T_{1}\left(P T_{1}\right)^{n-1} y_{n}\right) \\
y_{n}=P\left(\left(1-\beta_{n}\right) x_{n}+\beta_{n} T_{2}\left(P T_{2}\right)^{n-1} x_{n}\right), n \geq 1
\end{array}\right.
$$

If $\mathrm{T}$ is a self map, then $\mathrm{P}$ becomes the identity map so that (1.6) reduces to the Ishikawa-like iteration scheme without errors [9] involving two asymptotically nonexpansive self mappings. After that Chidume and Bashir ali 3 introduced a new iteration process for approximating common fixed points for finite families of nonself asymptotically nonexpansive mappings which is defined as follows:

$$
\left\{\begin{array}{l}
x_{1} \in K \\
x_{n+1}=P\left[\left(1-\alpha_{1 n}\right) x_{n}+\alpha_{1 n} T_{1}\left(P_{1}\right)^{n-1} y_{n+r-2}\right] \\
y_{n+r-2}=P\left[\left(1-\alpha_{2 n}\right) x_{n}+\alpha_{2 n} T_{2}\left(P T_{2}\right)^{n-1} y_{n+r-3}\right] \\
\cdot \\
\cdot \\
y_{n}=P\left[\left(1-\alpha_{m n}\right) x_{n}+\alpha_{m n} T_{m}\left(P_{m}\right)^{n-1} x_{n}\right]
\end{array}\right.
$$

Very recently Yang [20] introduced and studied a modified multistep iteration for a finite family of nonself asymptotically nonexpensive mappings and discuss their convergences which is defined as follows. 
For a given $x_{1} \in K$ and $n \geq 1$, compute the iterative sequences $\left\{x_{n}\right\},\left\{y_{n}\right\}, \ldots . .,\left\{y_{n+r-2}\right\}$ defined by

$$
\left\{\begin{aligned}
& y_{n}=P\left[\left(1-a_{n r}\right) x_{n}+a_{n r} T_{r}\left(P T_{r}\right)^{n-1} x_{n}\right] \\
& y_{n+1}= P\left[\left(1-a_{n(r-1)}-b_{n(r-1)}\right) x_{n}+a_{n(r-1)} T_{r-1}\left(P T_{r-1}\right)^{n-1} y_{n}\right. \\
&\left.+b_{n(r-1)} T_{r-1}\left(P T_{r-1}\right)^{n-1} x_{n}\right] \\
& y_{n+2}= P\left[\left(1-a_{n(r-2)}-b_{n(r-2)}\right) x_{n}+a_{n(r-2)} T_{r-2}\left(P T_{r-2}\right)^{n-1} y_{n+1}\right. \\
&\left.+b_{n(r-2)} T_{r-2}\left(P T_{r-2}\right)^{n-1} y_{n}\right] \\
& \\
& \cdot \\
& y_{n+r-2}= P\left[\left(1-a_{n 2}-b_{n 2}\right) x_{n}+a_{n 2} T_{2}\left(P T_{2}\right)^{n-1} y_{n+r-3}\right. \\
&\left.+b_{n 2} T_{2}\left(P T_{2}\right)^{n-1} y_{n+r-4}\right] \\
& x_{n+1}= P\left[\left(1-a_{n 1}-b_{n 1}\right) x_{n}+a_{n 1} T_{1}\left(P T_{1}\right)^{n-1} y_{n+r-2}\right. \\
&\left.+b_{n 1} T_{1}\left(P T_{1}\right)^{n-1} y_{n+r-3}\right]
\end{aligned}\right.
$$

where $\left\{a_{n i}\right\},\left\{b_{n i}\right\},\left\{1-a_{n i}-b_{n i}\right\}$ are appropriate real sequences in $[0,1]$ for $i \in I$ where $I=$ $\{1,2, \ldots . ., r\}$. Motivated by these facts we have introduced and studied a new type of multistep iterative process with errors which is defined as follows:

Let $E$ be a normed space, $K$ be a nonempty convex subset of $E$ which is also a nonexpansive retract of $E$. Let $T_{i}: K \rightarrow E(i \in I=\{1,2, \ldots, r\})$ be given nonself asymptotically nonexpansive mappings with sequences $\left\{k_{n}^{i}\right\} \subset[1, \infty)$ with $\lim _{n \rightarrow \infty} k_{n}^{i}=1$ for $i \in I$. Then for a given $x_{1} \in K$ and $n \geq 1$, compute the iterative sequences $\left\{x_{n}\right\},\left\{y_{n}\right\}, \ldots . .,\left\{y_{n+r-2}\right\}$ defined by

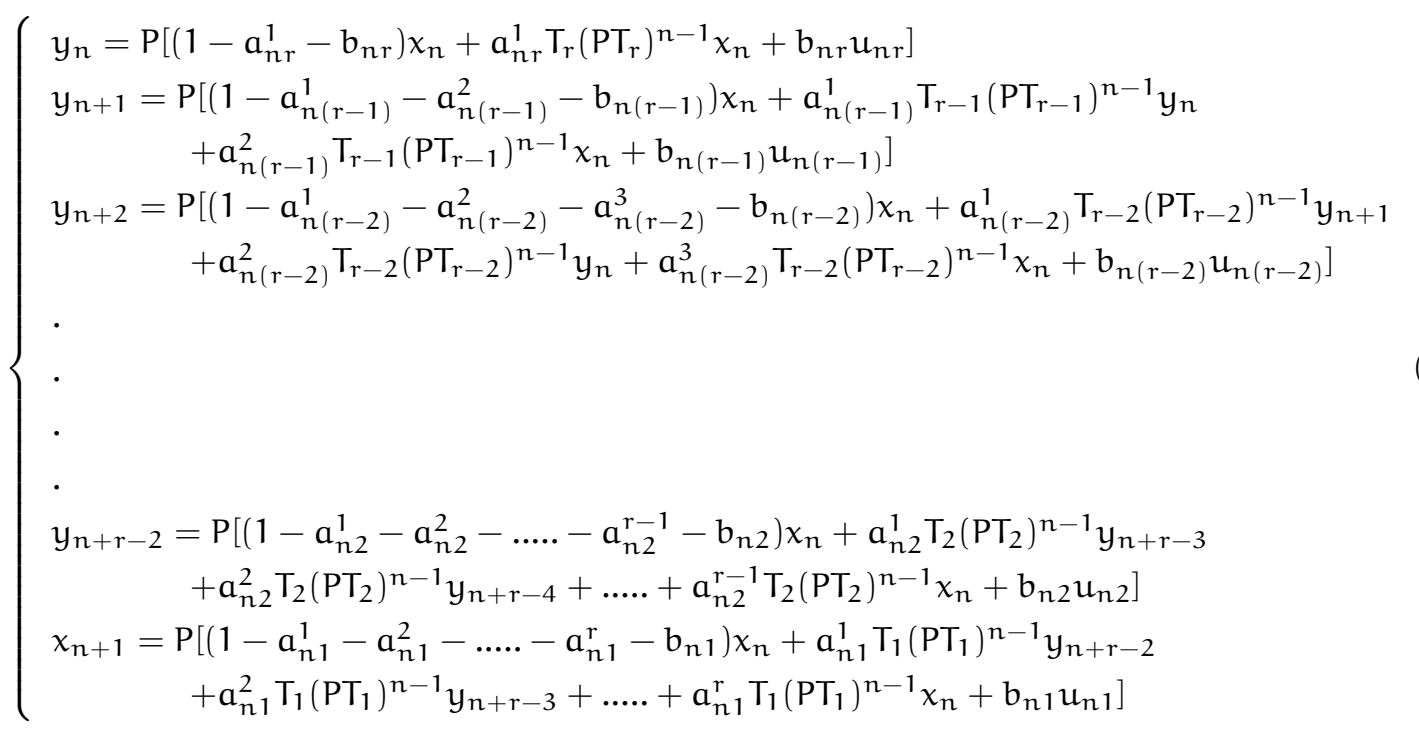

where $\left\{a_{n j}^{k}\right\},\left\{b_{n j}\right\},\left\{1-\sum_{k=1}^{r-j+1} a_{n j}^{k}-b_{n j}\right\}$ are appropriate real sequences in $[0,1]$ for $j \in I$ and $k \in\{1, \ldots, r-j+1\}$ and $\left\{u_{n j}\right\}$ are bounded sequences in $K$ for $j \in I$. The iterative sequence 
(1.9) is called the new modified multistep iteration for a finite family of nonself asymptotically nonexpansive mappings.

The iterative sequence (1.9) can be written as in the compact form

$$
\begin{aligned}
y_{n+r-j}= & P\left[\left(1-\sum_{k=1}^{r-j+1} a_{n j}^{k}-b_{n j}\right) x_{n}+\sum_{k=1}^{r-j} a_{n j}^{k} T_{j}\left(P T_{j}\right)^{n-1} y_{n+r-j-k}+\right. \\
& \left.a_{n j}^{r-j+1} T_{j}\left(P T_{j}\right)^{n-1} x_{n}+b_{n j} u_{n j}\right]
\end{aligned}
$$

where $j \in I$ and $x_{n+1}=y_{n+r-1}$.

As an illustration, for $r=3$, [1.9) reduces to the new modified three-step iteration with errors:

$$
\left\{\begin{aligned}
& y_{n}=P\left[\left(1-a_{n 3}^{1}-b_{n 3}\right) x_{n}+a_{n 3}^{1} T_{3}\left(P T_{3}\right)^{n-1} x_{n}+b_{n 3} u_{n 3}\right] \\
& y_{n+1}= P\left[\left(1-a_{n 2}^{1}-a_{n 2}^{2}-b_{n 2}\right) x_{n}+a_{n 2}^{1} T_{2}\left(P T_{2}\right)^{n-1} y_{n}\right. \\
&\left.+a_{n 2}^{2} T_{2}\left(P T_{2}\right)^{n-1} x_{n}+b_{n 2} u_{n 2}\right] \\
& x_{n+1}= P\left[\left(1-a_{n 1}^{1}-a_{n 1}^{2}-a_{n 1}^{3}-b_{n 1}\right) x_{n}+a_{n 1}^{1} T_{1}\left(P T_{1}\right)^{n-1} y_{n+1}\right. \\
&\left.+a_{n 1}^{2} T_{1}\left(P T_{1}\right)^{n-1} y_{n}+a_{n 1}^{3} T_{1}\left(P T_{1}\right)^{n-1} x_{n}+b_{n 1} u_{n 1}\right]
\end{aligned}\right.
$$

where $\left\{a_{\mathfrak{n} j}^{k}\right\},\left\{b_{n j}\right\},\left\{1-\sum_{k=1}^{3-j+1} a_{\mathfrak{n} j}^{k}-b_{\mathfrak{n} j}\right\}$ are appropriate real sequences in $[0,1]$ for $j \in\{1,2,3\}$ and $k \in\{1, \ldots, 3-j+1\}$ and $\left\{u_{n j}\right\}$ are bounded sequences in $K$ for $j \in\{1,2,3\}$.

For $a_{n j}^{k}=0$ for all $j \in\{1,2, \ldots, r-2\}$ and $k \in\{3,4, \ldots, r-j+1\}$ and $b_{n j}=0$ for all $j \in I$, (1.9) reduces to the iteration (1.8). Again if $a_{n j}^{k}=0$ for all $j \in\{1,2, \ldots, r-2, r-1\}$ and $k=\{2,3,4, \ldots, r-j+1\}$ and $b_{n j}=0$ for all $j \in I$, then (1.9) reduces to the iteration (1.7).

Next we recall the following definitions and results.

Let $E$ be a real normed linear space. The modulus of convexity of $E$ is a function $\delta_{E}:(0,2] \rightarrow[0,1]$ defined by

$$
\delta_{\mathrm{E}}(\epsilon)=\inf \left\{1-\left\|\frac{1}{2}(x+y)\right\|:\|x\|=1,\|y\|=1, \epsilon=\|x-y\|\right\} .
$$

$E$ is called uniformly convex if and only if $\delta_{E}(\epsilon)>0$ for all $\epsilon \in(0,2]$. The norm of $E$ is said to be Frèchet differentiable if for each $x \in E$ with $\|x\|=1$ the $\operatorname{limit}_{\lim } \lim _{t \rightarrow 0} \frac{\|x+t y\|-\|x\|}{t}$ exists and is attained uniformly for $y$ with $\|y\|=1$ and in this case it has been shown that in [18, that

$$
<\mathrm{h}, \mathrm{J}(\mathrm{x})>+\frac{1}{2}\|\mathrm{x}\|^{2} \leq \frac{1}{2}\|\mathrm{x}+\mathrm{h}\|^{2} \leq<\mathrm{h}, \mathrm{J}(\mathrm{x})>+\frac{1}{2}\|\mathrm{x}\|^{2}+\mathrm{b}(\|\mathrm{h}\|)
$$

for all $x, h \in E$ where $J$ is the Frèchet derivative of the functional $\|\cdot\|^{2}$ at $x \in E,\langle.,$.$\rangle is the$ pairing between $E$ and $E^{\star}$ and $b$ is a function defined on $[0, \infty)$ such that $\lim _{t \rightarrow 0} \frac{b(t)}{t}=0$. A Banach space $E$ is said to satisfy Opial's condition [12] if $x_{n} \rightarrow x$ and $x \neq y$ imply

$$
\limsup _{n \rightarrow \infty}\left\|x_{n}-x\right\|<\limsup _{n \rightarrow \infty}\left\|x_{n}-y\right\| .
$$

A Banach space $E$ is said to satisfy Kadec-Klee property, if for every sequence $\left\{x_{n}\right\} \in E, x_{n} \rightarrow x$ and $\left\|x_{n}\right\| \rightarrow\|x\|$ together imply that $x_{n} \rightarrow x$ as $n \rightarrow \infty$. There are uniformly convex Banach spaces which have neither a Frèchet differentiable norm nor satisfy Opial's property but their dual does have the Kadec-Klee property (see [6, [10]). 
Lemma 1.1. (18, Lemma1) Let $\left\{a_{n}\right\},\left\{b_{n}\right\}$ and $\left\{\delta_{n}\right\}$ be sequences of nonnegative real numbers satisfying the inequality

$$
a_{n+1} \leq\left(1+\delta_{n}\right) a_{n}+b_{n}, \forall n \geq 1 .
$$

If $\sum_{n=1}^{\infty} \delta_{n}<\infty$ and $\sum_{n=1}^{\infty} b_{n}<\infty$, then

(i) $\lim _{n \rightarrow \infty} a_{n}$ exists,

(ii) $\lim _{n \rightarrow \infty} a_{n}=0$ whenever $\liminf _{n \rightarrow \infty} a_{n}=0$.

Lemma 1.2. (21], Theorem2) Let $p>1$ and $r>0$ be two fixed real numbers. Then a Banach space $E$ is uniformly convex if and only if there exists a continuous strictly increasing convex function $g:[0, \infty) \rightarrow[0, \infty)$ with $g(0)=0$ such that

$$
\|\lambda x+(1-\lambda) y\|^{p} \leq \lambda\|x\|^{p}+(1-\lambda)\|y\|^{p}-\omega_{p}(\lambda) g(\|x-y\|)
$$

for all $x, y \in B_{r}(0)=\{x \in E:\|x\| \leq r\}$ and $\lambda \in[0,1]$ where $\omega_{p}(\lambda)=\lambda^{p}(1-\lambda)+\lambda(1-\lambda)^{p}$.

Lemma 1.3. ([5, Lemma1.4) Let $E$ be a uniformly convex Banach space and $B_{r}=\{x \in E:\|x\| \leq r\}$, $r>0$. Then there exist a continuous, strictly increasing and convex function $g:[0, \infty) \rightarrow[0, \infty)$, $g(0)=0$ such that

$$
\|\lambda x+\beta y+\gamma z\|^{2} \leq \lambda\|x\|^{2}+\beta\|y\|^{2}+\gamma\|z\|^{2}-\lambda \beta g(\|x-y\|)
$$

for all $x, y, z \in B_{r}$ and all $\lambda, \beta, \gamma \in[0,1]$ with $\lambda+\beta+\gamma=1$.

By using Lemma 1.2 and Lemma 1.3 we can easily prove the following Lemma:

Lemma 1.4. Let $\mathrm{E}$ be a uniformly convex Banach space and $\mathrm{B}_{\mathrm{r}}=\{x \in \mathrm{E}:\|x\| \leq \mathrm{r}\}, \mathrm{r}>0$. Then there exists a continuous, strictly increasing and convex function $g:[0, \infty) \rightarrow[0, \infty), g(0)=0$ such that

$$
\left\|\lambda_{1} x_{1}+\lambda_{2} x_{2}+\ldots+\lambda_{n} x_{n}\right\|^{2} \leq \lambda_{1}\left\|x_{1}\right\|^{2}+\lambda_{2}\left\|x_{2}\right\|^{2}+\ldots+\lambda_{n}\left\|x_{n}\right\|^{2}-\lambda_{1} \lambda_{2} g\left(\left\|x_{1}-x_{2}\right\|\right)
$$

for all $x_{i} \in B_{r}$ and all $\lambda_{i} \in[0,1]$ for all $i=1,2, \ldots, n$ with $\sum_{i=1}^{n} \lambda_{i}=1$.

Proof: The Lemma is true for $n=2$ since for $n=2$ using Lemma 1.2 we get

$$
\left\|\lambda_{1} x_{1}+\lambda_{2} x_{2}\right\|^{2} \leq \lambda_{1}\left\|x_{1}\right\|^{2}+\lambda_{2}\left\|x_{2}\right\|^{2}-\omega_{2}\left(\lambda_{1}\right) g\left(\left\|x_{1}-x_{2}\right\|\right)
$$

where $\omega_{2}\left(\lambda_{1}\right)=\lambda_{1}^{2}\left(1-\lambda_{1}\right)+\lambda_{1}\left(1-\lambda_{1}\right)^{2}=\lambda_{1}^{2} \lambda_{2}+\lambda_{1} \lambda_{2}^{2}=\lambda_{1} \lambda_{2}\left(\lambda_{1}+\lambda_{2}\right)=\lambda_{1} \lambda_{2}$. Also by Lemma 1.3 we see that this Lemma is true for $n=3$. Now let the Lemma is true for $n=m$. Now

$$
\begin{aligned}
& \left\|\lambda_{1} x_{1}+\lambda_{2} x_{2}+\ldots+\lambda_{m} x_{m}+\lambda_{m+1} x_{m+1}\right\|^{2} \\
= & \| \lambda_{1} x_{1}+\lambda_{2} x_{2}+\ldots+\lambda_{m-1} x_{m-1}+ \\
& \left(1-\lambda_{1}-\lambda_{2}-\ldots . .-\lambda_{m-1}\right)\left(\frac{\lambda_{m}}{1-\lambda_{1}-\lambda_{2}-\ldots . \lambda_{m-1}} x_{m}+\frac{\lambda_{m+1}}{1-\lambda_{1}-\lambda_{2}-\ldots . .-\lambda_{m-1}} x_{m+1}\right) \|^{2} .
\end{aligned}
$$


By using the above inequality,

$$
\begin{aligned}
& \left\|\lambda_{1} x_{1}+\lambda_{2} x_{2}+\ldots+\lambda_{m} x_{m}+\lambda_{m+1} x_{m+1}\right\|^{2} \\
= & \| \lambda_{1} x_{1}+\lambda_{2} x_{2}+\ldots+\lambda_{m-1} x_{m-1} \\
& \left(1-\lambda_{1}-\lambda_{2}-\ldots .+\lambda_{m-1}\right)\left(\frac{\lambda_{m}}{1-\lambda_{1}-\lambda_{2}-\ldots .-\lambda_{m-1}} x_{m}+\frac{\lambda_{m+1}}{1-\lambda_{1}-\lambda_{2}-\ldots . .-\lambda_{m-1}} x_{m+1}\right) \|^{2} \\
\leq & \lambda_{1}\left\|x_{1}\right\|^{2}+\lambda_{2}\left\|x_{2}\right\|^{2}+\ldots+\lambda_{m-1}\left\|x_{m-1}\right\|^{2}+ \\
& \left(1-\lambda_{1}-\lambda_{2}-\ldots . .-\lambda_{m-1}\right)\left\|\frac{\lambda_{m+1}}{1-\lambda_{1}-\lambda_{2}-\ldots . .-\lambda_{m-1}} x_{m}+\frac{\lambda_{m}}{1-\lambda_{1}-\lambda_{2}-\ldots . \lambda_{m-1}} x_{m+1}\right\|^{2} \\
& -\lambda_{1} \lambda_{2} g\left(\left\|x_{1}-x_{2}\right\|\right) .
\end{aligned}
$$

Since $\lambda_{1}+\lambda_{2}+\ldots+\lambda_{m}+\lambda_{m+1}=1$, so

$$
\frac{\lambda_{m}}{1-\lambda_{1}-\lambda_{2}-\ldots . .-\lambda_{m-1}}+\frac{\lambda_{m+1}}{1-\lambda_{1}-\lambda_{2}-\ldots . .-\lambda_{m-1}}=\frac{\lambda_{m}+\lambda_{m+1}}{1-\lambda_{1}-\lambda_{2}-\ldots . \lambda_{m-1}}=1 .
$$

Then from Lemma 1.2 we get that

$$
\begin{aligned}
& \left\|\frac{\lambda_{m}}{1-\lambda_{1}-\lambda_{2}-\ldots . .-\lambda_{m-1}} x_{m}+\frac{\lambda_{m+1}}{1-\lambda_{1}-\lambda_{2}-\ldots . .-\lambda_{m-1}} x_{m+1}\right\|^{2} \\
\leq & \frac{\lambda_{m}}{1-\lambda_{1}-\lambda_{2}-\ldots . \lambda_{m-1}}\left\|x_{m}\right\|^{2}+\frac{\lambda_{m+1}}{1-\lambda_{1}-\lambda_{2}-\ldots . .-\lambda_{m-1}}\left\|x_{m+1}\right\|^{2} \\
& -\omega_{2}\left(\frac{\lambda_{m}}{1-\lambda_{1}-\lambda_{2}-\ldots . .-\lambda_{m-1}}\right) g\left(\left\|x_{1}-x_{2}\right\|\right) .
\end{aligned}
$$

Now,

$$
\begin{aligned}
& \omega_{2}\left(\frac{\lambda_{m}}{1-\lambda_{1}-\lambda_{2}-\ldots . \lambda_{m-1}}\right) \\
& =\left(\frac{\lambda_{m}}{1-\lambda_{1}-\lambda_{2}-\ldots . \lambda_{m-1}}\right)^{2}\left(1-\frac{\lambda_{m}}{1-\lambda_{1}-\lambda_{2}-\ldots . .-\lambda_{m-1}}\right) \\
& +\frac{\lambda_{m}}{1-\lambda_{1}-\lambda_{2}-\ldots . .-\lambda_{m-1}}\left(1-\frac{\lambda_{m}}{1-\lambda_{1}-\lambda_{2}-\ldots . .-\lambda_{m-1}}\right)^{2} \\
& =\frac{\lambda_{m}}{1-\lambda_{1}-\lambda_{2}-\ldots . \lambda_{m-1}} \cdot \frac{\lambda_{m+1}}{1-\lambda_{1}-\lambda_{2}-\ldots . .-\lambda_{m-1}} \\
& \left(\frac{\lambda_{m}}{1-\lambda_{1}-\lambda_{2}-\ldots . \lambda_{m-1}}+\frac{\lambda_{m+1}}{1-\lambda_{1}-\lambda_{2}-\ldots . .-\lambda_{m-1}}\right) \\
& =\frac{\lambda_{m} \lambda_{m+1}}{\left(1-\lambda_{1}-\lambda_{2}-\ldots . \lambda_{m-1}\right)^{2}} \geq 0 \text {. }
\end{aligned}
$$

Therefore from above we have

$$
\begin{gathered}
\left\|\frac{\lambda_{m}}{1-\lambda_{1}-\lambda_{2}-\ldots . \lambda_{m-1}} x_{m}+\frac{\lambda_{m+1}}{1-\lambda_{1}-\lambda_{2}-\ldots .-\lambda_{m-1}} x_{m+1}\right\|^{2} \\
\leq \frac{\lambda_{m}}{1-\lambda_{1}-\lambda_{2}-\ldots . .-\lambda_{m-1}}\left\|x_{m}\right\|^{2}+\frac{\lambda_{m+1}}{1-\lambda_{1}-\lambda_{2}-\ldots . .-\lambda_{m-1}}\left\|x_{m+1}\right\|^{2} .
\end{gathered}
$$


So finally we get that

$$
\begin{aligned}
& \left\|\lambda_{1} x_{1}+\lambda_{2} x_{2}+\ldots+\lambda_{m} x_{m}+\lambda_{m+1} x_{m+1}\right\|^{2} \\
= & \| \lambda_{1} x_{1}+\lambda_{2} x_{2}+\ldots+\lambda_{m-1} x_{m-1}+ \\
& \left(1-\lambda_{1}-\lambda_{2}-\ldots . . \lambda_{m-1}\right)\left(\frac{\lambda_{m}}{1-\lambda_{1}-\lambda_{2}-\ldots . .-\lambda_{m-1}} x_{m}+\frac{\lambda_{m+1}}{1-\lambda_{1}-\lambda_{2}-\ldots .-\lambda_{m-1}} x_{m+1}\right) \|^{2} \\
\leq & \lambda_{1}\left\|x_{1}\right\|^{2}+\lambda_{2}\left\|x_{2}\right\|^{2}+\ldots+\lambda_{m-1}\left\|x_{m-1}\right\|^{2}+ \\
& \left(1-\lambda_{1}-\lambda_{2}-\ldots . .-\lambda_{m-1}\right)\left(\frac{\lambda_{m}}{1-\lambda_{1}-\lambda_{2}-\ldots . .-\lambda_{m-1}}\left\|x_{m}\right\|^{2}+\right. \\
& \left.\frac{\lambda_{m+1}}{1-\lambda_{1}-\lambda_{2}-\ldots .-\lambda_{m-1}}\left\|x_{m+1}\right\|^{2}\right)-\lambda_{1} \lambda_{2} g\left(\left\|x_{1}-x_{2}\right\|\right) \\
= & \lambda_{1}\left\|x_{1}\right\|^{2}+\lambda_{2}\left\|x_{2}\right\|^{2}+\ldots+\lambda_{m-1}\left\|x_{m-1}\right\|^{2}+\lambda_{m}\left\|x_{m}\right\|^{2}+\lambda_{m+1}\left\|x_{m+1}\right\|^{2}-\lambda_{1} \lambda_{2} g\left(\left\|x_{1}-x_{2}\right\|\right) .
\end{aligned}
$$

Hence the Lemma is true for $n=m+1$. Thus, by induction, the Lemma is true for all $n \geq 2$. This completes the proof of the Lemma.

Lemma 1.5. (2, Theorem3.4) Let $\mathrm{E}$ be a real uniformly Banach space and $\mathrm{K}$ be a nonempty closed convex subset of $\mathrm{E}$ and $\mathrm{T}: \mathrm{K} \rightarrow \mathrm{E}$ be asymptotically nonexpansive mapping with a sequence $\left\{k_{n}\right\} \subset[1, \infty)$ with $\lim _{n \rightarrow \infty} k_{n}=1$. Then $I-T$ is demiclosed at zero, i.e. if $\left\{x_{n}\right\}$ is a sequence in $K$ which converges weakly to $x$ and if the sequence $\left\{x_{n}-T x_{n}\right\}$ converges strongly to zero, then $x-\mathrm{T} x=0$.

Lemma 1.6. (10, Theorem2) Let $\mathrm{E}$ be a real reflexive Banach space such that $\mathrm{E}^{\star}$ has the KadecKlee property. Let $\left\{x_{n}\right\}$ be a bounded sequence in $E$ and $x^{\star}, y^{\star} \in \mathrm{w}_{w}\left(x_{n}\right)$ (weak $w$-limit set of $\left.\left\{x_{n}\right\}\right)$. Suppose $\lim _{n \rightarrow \infty}\left\|t x_{n}+(1-t) x^{\star}-y^{\star}\right\|$ exists for all $t \in[0,1]$. Then $x^{\star}=y^{\star}$.

Lemma 1.7. (1]) Let $\mathrm{E}$ be a uniformly convex Banach space $\mathrm{K}$ be a nonempty bounded closed convex subset of $E$. Then there exists a strictly increasing continuous convex function $\phi:[0, \infty) \rightarrow$ $[0, \infty)$ with $\phi(0)=0$ such that for any Lipschitzian mapping $\mathrm{T}: \mathrm{K} \rightarrow \mathrm{E}$ with the Lipschitz constant $\mathrm{L} \geq 1$ and for any $x, y \in K$ and $t \in[0,1]$ the following inequality holds:

$$
\|\mathrm{T}(\mathrm{t} x+(1-\mathrm{t}) \mathrm{y})-(\mathrm{tT} x+(1-\mathrm{t}) \mathrm{T} y)\| \leq \mathrm{L} \phi^{-1}\left(\|x-y\|-\mathrm{L}^{-1}\|\mathrm{~T} x-\mathrm{Ty}\|\right)
$$

The purpose of this paper is to introduce a new modified multi step iteration with errors for approximating common fixed points for finite families of nonself asymptotically nonexpansive mappings. We prove some strong and weak convergence theorems in real uniformly convex Banach spaces. More precisely we prove convergence theorems in a uniformly convex Banach space which satisfy Opial's condition or have Frèchet differentiable norm or whose duals have the Kadec-Klee property. Our results generalize some recent results.

\section{Main Results}

We begin this section with the following lemmas. 
Lemma 2.1. Let $\mathrm{E}$ be a real normed space and $\mathrm{K}$ be a nonempty subset of $\mathrm{E}$ which is also a nonexpansive retract of $E$. Let $T_{i}: K \rightarrow E(i \in I=\{1,2, \ldots, r\})$ be given nonself asymptotically nonexpansive mappings with sequences $\left\{k_{n}^{i}\right\} \subset[1, \infty)$ with $\sum_{n=1}^{\infty}\left(k_{n}^{i}-1\right)<\infty$ for $i \in$ I. Let $\left\{x_{n}\right\}$ be defined by (1.9) with $\sum_{n=1}^{\infty} b_{n i}<\infty$ for $i \in$ I. If $F=\bigcap_{i=1}^{r} F\left(T_{i}\right) \neq \emptyset$, then $\lim _{n \rightarrow \infty}\left\|x_{n}-q\right\|$ exists for all $\mathrm{q} \in \mathrm{F}$.

Proof: Let $\mathrm{q} \in$ F. For each $\mathrm{n} \geq 1$, let $\mathrm{k}_{\mathrm{n}}=\max \left\{k_{n}^{1}, \mathrm{k}_{n}^{2}, \ldots \ldots \ldots . ., \mathrm{k}_{n}^{r}\right\}$ so that $\left\{k_{n}\right\} \subset$ $[1, \infty)$ with $\sum_{n=1}^{\infty}\left(k_{n}-1\right)<\infty$. Since $\left\{u_{n i}\right\}$ are bounded sequences in $K$ for $i \in I$, let $M=$ $\sup _{n \geq 1, i=1,2, \ldots, r}\left\|u_{n i}-q\right\|$. From (1.9) we get

$$
\begin{aligned}
\left\|y_{n}-q\right\| & =\left\|P\left(\left(1-a_{n r}^{1}-b_{n r}\right) x_{n}+a_{n r}^{1} T_{r}\left(P T_{r}\right)^{n-1} x_{n}+b_{n r} u_{n r}\right)-P q\right\| \\
& \leq\left\|\left(1-a_{n r}^{1}-b_{n r}\right)\left(x_{n}-q\right)+a_{n r}^{1}\left(T_{r}\left(P T_{r}\right)^{n-1} x_{n}-q\right)+b_{n r}\left(u_{n r}-q\right)\right\| \\
& \leq\left(1-a_{n r}^{1}-b_{n r}\right)\left\|x_{n}-q\right\|+a_{n r}^{1}\left\|T_{r}\left(P T_{r}\right)^{n-1} x_{n}-q\right\|+b_{n r}\left\|u_{n r}-q\right\| \\
& \leq\left(1-a_{n r}^{1}\right)\left\|x_{n}-q\right\|+a_{n r}^{1} k_{n}\left\|x_{n}-q\right\|+b_{n r} M \\
& \leq k_{n}\left\|x_{n}-q\right\|+b_{n r} M \\
& =k_{n}\left\|x_{n}-q\right\|+\sigma_{n}^{1}
\end{aligned}
$$

where $\sigma_{n}^{1}=b_{n r} M$. By the given condition we get that $\sum_{n=1}^{\infty} \sigma_{n}^{1}<\infty$. Also from (1.9) and (2.1) we have

$$
\begin{aligned}
\left\|y_{n+1}-q\right\|= & \| P\left(\left(1-a_{n(r-1)}^{1}-a_{n(r-1)}^{2}-b_{n(r-1)}\right) x_{n}+a_{n(r-1)}^{1} T_{r-1}\left(P T_{r-1}\right)^{n-1} y_{n}+\right. \\
& \left.a_{n(r-1)}^{2} T_{r-1}\left(P T_{r-1}\right)^{n-1} x_{n}+b_{n(r-1)} u_{n(r-1)}\right)-P q \| \\
\leq & \|\left(1-a_{n(r-1)}^{1}-a_{n(r-1)}^{2}-b_{n(r-1)}\right)\left(x_{n}-q\right)+a_{n(r-1)}^{1}\left(T_{r-1}\left(P T_{r-1}\right)^{n-1} y_{n}-q\right) \\
& +a_{n(r-1)}^{2}\left(T_{r-1}\left(P T_{r-1}\right)^{n-1} x_{n}-q\right)+b_{n(r-1)}\left(u_{n(r-1)}-q\right) \| \\
\leq & \left(1-a_{n(r-1)}^{1}-a_{n(r-1)}^{2}-b_{n(r-1)}\right)\left\|x_{n}-q\right\|+a_{n(r-1)}^{1}\left\|T_{r-1}\left(P T_{r-1}\right)^{n-1} y_{n}-q\right\| \\
& +a_{n(r-1)}^{2}\left\|T_{r-1}\left(P T_{r-1}\right)^{n-1} x_{n}-q\right\|+b_{n(r-1)}\left\|u_{n(r-1)}-q\right\| \\
\leq & \left(1-a_{n(r-1)}^{1}-a_{n(r-1)}^{2}\right)\left\|x_{n}-q\right\|+a_{n(r-1)}^{1} k_{n}\left\|y_{n}-q\right\| \\
& +a_{n(r-1)}^{2} k_{n}\left\|x_{n}-q\right\|+b_{n(r-1)} M \\
\leq & \left(1-a_{n(r-1)}^{1}-a_{n(r-1)}^{2}\right)\left\|x_{n}-q\right\|+a_{n(r-1)}^{1} k_{n}\left[k_{n}\left\|x_{n}-q\right\|+b_{n r} M\right] \\
& +a_{n(r-1)}^{2} k_{n}\left\|x_{n}-q\right\|+b_{n(r-1)} M \\
\leq & {\left[1+a_{n(r-1)}^{1}\left(k_{n}^{2}-1\right)+a_{n(r-1)}^{2}\left(k_{n}-1\right)\right]\left\|x_{n}-q\right\|+a_{n(r-1)}^{1} k_{n} b_{n r} M+} \\
& b_{n(r-1)} M \\
\leq & k_{n}^{2}\left\|x_{n}-q\right\|+k_{n} b_{n r} M+b_{n(r-1)} M \\
= & k_{n}^{2}\left\|x_{n}-q\right\|+\sigma_{n}^{2}
\end{aligned}
$$

where $\sigma_{n}^{2}=k_{n} b_{n r} M+b_{n(r-1)} M$. By the given condition we get that $\sum_{n=1}^{\infty} \sigma_{n}^{2}<\infty$. Also from 
(1.9) and (2.2) we have

$$
\begin{aligned}
& \left\|y_{n+2}-q\right\|= \\
& \| P\left(\left(1-a_{n(r-2)}^{1}-a_{n(r-2)}^{2}-a_{n(r-2)}^{3}-b_{n(r-2)}\right) x_{n}+a_{n(r-2)}^{1} T_{r-2}\left(P T_{r-2}\right)^{n-1} y_{n+1}\right. \\
& \left.+a_{n(r-2)}^{2} T_{r-2}\left(P T_{r-2}\right)^{n-1} y_{n}+a_{n(r-2)}^{3} T_{r-2}\left(P T_{r-2}\right)^{n-1} x_{n}+b_{n(r-2)} u_{n(r-2)}\right)-P q \| \\
\leq & \|\left(1-a_{n(r-2)}^{1}-a_{n(r-2)}^{2}-a_{n(r-2)}^{3}-b_{n(r-2)}\right)\left(x_{n}-q\right)+a_{n(r-2)}^{1}\left(T_{r-2}\left(P T_{r-2}\right)^{n-1} y_{n+1}-q\right) \\
& +a_{n(r-2)}^{2}\left(T_{r-2}\left(P T_{r-2}\right)^{n-1} y_{n}-q\right)+a_{n(r-2)}^{3}\left(T_{r-2}\left(P T_{r-2}\right)^{n-1} x_{n}-q\right) \\
& +b_{n(r-2)}\left(u_{n(r-2)}-q\right) \| \\
\leq & \left(1-a_{n(r-2)}^{1}-a_{n(r-2)}^{2}-a_{n(r-2)}^{3}-b_{n(r-2)}\right)\left\|x_{n}-q\right\|+a_{n(r-2)}^{1}\left\|T_{r-2}\left(P T_{r-2}\right)^{n-1} y_{n+1}-q\right\| \\
& +a_{n(r-2)}^{2}\left\|T_{r-2}\left(P T_{r-2}\right)^{n-1} y_{n}-q\right\|+a_{n(r-2)}^{3}\left\|T_{r-2}\left(P T_{r-2}\right)^{n-1} x_{n}-q\right\| \\
& +b_{n(r-2)}\left\|u_{n(r-2)}-q\right\| \\
\leq & \left(1-a_{n(r-2)}^{1}-a_{n(r-2)}^{2}-a_{n(r-2)}^{3}\right)\left\|x_{n}-q\right\|+a_{n(r-2)}^{1} k_{n}\left\|y_{n+1}-q\right\| \\
& +a_{n(r-2)}^{2} k_{n}\left\|y_{n}-q\right\|+a_{n(r-2)}^{3} k_{n}\left\|x_{n}-q\right\|+b_{n(r-2)} M \\
\leq & \left(1-a_{n(r-2)}^{1}-a_{n(r-2)}^{2}-a_{n(r-2)}^{3}\right)\left\|x_{n}-q\right\|+a_{n(r-2)}^{1} k_{n}\left[k_{n}^{2}\left\|x_{n}-q\right\|\right. \\
& \left.+k_{n} b_{n r} M+b_{n(r-1)} M\right]+a_{n(r-2)}^{2} k_{n}\left[k_{n}\left\|x_{n}-q\right\|+b_{n r} M\right] \\
& +a_{n(r-2)}^{3} k_{n}\left\|x_{n}-q\right\|+b_{n(r-2)} M \\
\leq & k_{n}^{3}\left\|x_{n}-q\right\|+\sigma_{n}^{3}
\end{aligned}
$$

where $\sigma_{n}^{3}=k_{n}^{2} b_{n r} M+k_{n} b_{n r} M+k_{n} b_{n(r-1)} M+b_{n(r-2)} M$. By the given condition we get that $\sum_{n=1}^{\infty} \sigma_{n}^{3}<\infty$. In general after $(j+1)$ steps we get

$$
\left\|y_{n+j}-q\right\| \leq k_{n}^{j+1}\left\|x_{n}-q\right\|+\sigma_{n}^{j+1}
$$

for $j=0,1, \ldots, r-2$ and $\left\{\sigma_{n}^{j+1}\right\}$ is a nonnegative real sequence such that $\sum_{n=1}^{\infty} \sigma_{n}^{j+1}<\infty$ for $j=0,1, \ldots, r-2$. Therefore it follows from (1.9) and [2.4] that

$$
\left\|x_{n+1}-q\right\| \leq k_{n}^{r}\left\|x_{n}-q\right\|+\sigma_{n}^{r}=\left[1+\left(k_{n}^{r}-1\right)\right]\left\|x_{n}-q\right\|+\sigma_{n}^{r}
$$

where $\left\{\sigma_{n}^{r}\right\}$ is a nonnegative real sequence such that $\sum_{n=1}^{\infty} \sigma_{n}^{r}<\infty$. Since $0 \leq t^{r}-1 \leq r t^{r-1}(t-1)$ for all $t \geq 1$, so $0 \leq k_{n}^{r}-1 \leq r k_{n}^{r-1}\left(k_{n}-1\right)$. Since $\sum_{n=1}^{\infty}\left(k_{n}-1\right)<\infty$ so $\left\{k_{n}\right\}$ is bounded, $k_{n} \in\left[1, M^{\prime}\right]$ for some $M^{\prime}>0$. So $\sum_{n=1}^{\infty}\left(k_{n}^{r}-1\right)<\infty$. Thus by Lemma 1.1]we get $\lim _{n \rightarrow \infty}\left\|x_{n}-q\right\|$ exists for all $q \in F . \diamond$

Lemma 2.2. Let $\mathrm{E}$ be a uniformly convex Banach space and $\mathrm{K}$ be a nonempty closed convex subset of $E$ which is also a nonexpansive retract of $E$. Let $T_{i}: K \rightarrow E(i \in I=\{1,2, \ldots, r\})$ be given nonself asymptotically nonexpansive mappings with sequences $\left\{k_{n}^{i}\right\} \subset[1, \infty)$ with $\sum_{n=1}^{\infty}\left(k_{n}^{i}-1\right)<\infty$ for $i \in$ I. Let $\left\{x_{n}\right\}$ be defined by (1.9) with $\sum_{n=1}^{\infty} b_{n i}<\infty$ for $i \in$ I. If $F=\bigcap_{i=1}^{r} F\left(T_{i}\right) \neq \emptyset$, then the following results hold

(1) If $\liminf _{n \rightarrow \infty} a_{n k}^{1}>0$, for all $k<r$ and $0<\liminf _{n \rightarrow \infty} a_{n r}^{1} \leq \limsup _{n \rightarrow \infty}\left(a_{n r}^{1}+b_{n r}\right)<1$ then $\lim _{n \rightarrow \infty}\left\|T_{r}\left(P T_{r}\right)^{n-1} x_{n}-x_{n}\right\|=0$. 
(2) If $0<\liminf _{n \rightarrow \infty} a_{n 1}^{1} \leq \limsup \sup _{n \rightarrow \infty}\left(\sum_{k=1}^{r} a_{n 1}^{k}+b_{n 1}\right)<1$ then $\lim _{n \rightarrow \infty} \| T_{1}\left(P T_{1}\right)^{n-1} y_{n+r-2}-$ $x_{n} \|=0$.

(3) If $\liminf _{n \rightarrow \infty} a_{n k}^{1}>0$, for all $k<j$ and $0<\liminf _{n \rightarrow \infty} a_{n j}^{1} \leq \limsup _{n \rightarrow \infty}\left(\sum_{m=1}^{r-j+1} a_{n j}^{m}+\right.$ $\left.b_{\mathfrak{n} j}\right)<1$, then $\lim _{n \rightarrow \infty}\left\|T_{j}\left(P T_{j}\right)^{n-1} y_{n+r-j-1}-x_{n}\right\|=0$ for $j=2,3, \ldots . ., r-1$.

Proof: Let $q \in F$. By Lemma 2.1 we have that $\lim _{n \rightarrow \infty}\left\|x_{n}-q\right\|$ exists for all $q \in F$. So $\left\{x_{n}-q\right\}$ is bounded in K. Since $\left\{k_{n}\right\}$ and $\left\{\sigma_{n}^{j+1}\right\}$ are bounded so it follows from (2.4) that $\left\{y_{n+j}-q\right\}$ are bounded for $j=0,1, \ldots, r-2$. Since $T_{j}$ is a nonself asymptotically nonexpansive mapping, we have

$$
\left\|T_{j}\left(P T_{j}\right)^{n-1} y_{n+r-j-1}-q\right\| \leq k_{n}^{j}\left\|y_{n+r-j-1}-q\right\|
$$

for $j=1, \ldots, r-1$. Therefore the sequences $\left\{T_{j}\left(P T_{j}\right)^{n-1} y_{n+r-j-1}-q\right\}$ are bounded for $j=1, \ldots, r-1$. Therefore there exists $\mathrm{D}>0$ such that $\mathrm{K} \subseteq \mathrm{B}_{\mathrm{D}}$. From [1.9] and Lemma 1.3 we get

$$
\begin{aligned}
\left\|y_{n}-q\right\|^{2}= & \left\|P\left(\left(1-a_{n r}^{1}-b_{n r}\right) x_{n}+a_{n r}^{1} T_{r}\left(P T_{r}\right)^{n-1} x_{n}+b_{n r} u_{n r}\right)-P q\right\|^{2} \\
\leq & \left\|\left(1-a_{n r}^{1}-b_{n r}\right)\left(x_{n}-q\right)+a_{n r}^{1}\left(T_{r}\left(P T_{r}\right)^{n-1} x_{n}-q\right)+b_{n r}\left(u_{n r}-q\right)\right\|^{2} \\
\leq & \left(1-a_{n r}^{1}-b_{n r}\right)\left\|x_{n}-q\right\|^{2}+a_{n r}^{1}\left\|T_{r}\left(P T_{r}\right)^{n-1} x_{n}-q\right\|^{2}+b_{n r}\left\|u_{n r}-q\right\|^{2}- \\
& \left(1-a_{n r}^{1}-b_{n r}\right) a_{n r}^{1} g_{1}\left(\left\|T_{r}\left(P T_{r}\right)^{n-1} x_{n}-x_{n}\right\|\right) \\
\leq & \left(1-a_{n r}^{1}\right)\left\|x_{n}-q\right\|^{2}+a_{n r}^{1} k_{n}^{2}\left\|x_{n}-q\right\|^{2}+b_{n r} M^{2}- \\
& \left(1-a_{n r}^{1}-b_{n r}\right) a_{n r}^{1} g_{1}\left(\left\|T_{r}\left(P T_{r}\right)^{n-1} x_{n}-x_{n}\right\|\right) \\
\leq & k_{n}^{2}\left\|x_{n}-q\right\|^{2}+\mu_{n}^{1}-a_{n r}^{1}\left(1-a_{n r}^{1}-b_{n r}\right) g_{1}\left(\left\|T_{r}\left(P T_{r}\right)^{n-1} x_{n}-x_{n}\right\|\right)
\end{aligned}
$$


where $\mu_{n}^{1}=b_{n r} M^{2}$ so that $\sum_{n=1}^{\infty} \mu_{n}^{1}<\infty$. From (1.9) and (2.6) and from Lemma 1.4 we get

$$
\begin{aligned}
\left\|y_{n+1}-q\right\|^{2}= & \| P\left[\left(1-a_{n(r-1)}^{1}-a_{n(r-1)}^{2}-b_{n(r-1)}\right) x_{n}+a_{n(r-1)}^{1} T_{r-1}\left(P T_{r-1}\right)^{n-1} y_{n}+\right. \\
& \left.a_{n(r-1)}^{2} T_{r-1}\left(P T_{r-1}\right)^{n-1} x_{n}+b_{n(r-1)} u_{n(r-1)}\right]-P q \|^{2} \\
\leq & \|\left(1-a_{n(r-1)}^{1}-a_{n(r-1)}^{2}-b_{n(r-1)}\right)\left(x_{n}-q\right)+a_{n(r-1)}^{1}\left(T_{r-1}\left(P T_{r-1}\right)^{n-1} y_{n}-q\right) \\
& +a_{n(r-1)}^{2}\left(T_{r-1}\left(P T_{r-1}\right)^{n-1} x_{n}-q\right)+b_{n(r-1)}\left(u_{n(r-1)}-q\right) \|^{2} \\
\leq & \left(1-a_{n(r-1)}^{1}-a_{n(r-1)}^{2}-b_{n(r-1)}\right)\left\|x_{n}-q\right\|^{2}+a_{n(r-1)}^{1}\left\|T_{r-1}\left(P T_{r-1}\right)^{n-1} y_{n}-q\right\|^{2} \\
& +a_{n(r-1)}^{2}\left\|T_{r-1}\left(P T_{r-1}\right)^{n-1} x_{n}-q\right\|^{2}+b_{n(r-1)}\left\|u_{n(r-1)}-q\right\|^{2} \\
& -a_{n(r-1)}^{1}\left(1-a_{n(r-1)}^{1}-a_{n(r-1)}^{2}-b_{n(r-1)}\right) g_{2}\left(\left\|T_{r-1}\left(P T_{r-1}\right)^{n-1} y_{n}-x_{n}\right\|\right) \\
\leq & \left(1-a_{n(r-1)}^{1}-a_{n(r-1)}^{2}-b_{n(r-1)}\right)\left\|x_{n}-q\right\|^{2}+a_{n(r-1)}^{1} k_{n}^{2}\left\|y_{n}-q\right\|^{2} \\
& +a_{n(r-1)}^{2} k_{n}^{2}\left\|x_{n}-q\right\|^{2}+b_{n(r-1)} M^{2} \\
& -a_{n(r-1)}^{1}\left(1-a_{n(r-1)}^{1}-a_{n(r-1)}^{2}-b_{n(r-1)}\right) g_{2}\left(\left\|T_{r-1}\left(P T_{r-1}\right)^{n-1} y_{n}-x_{n}\right\|\right) \\
\leq & \left(1-a_{n(r-1)}^{1}-a_{n(r-1)}^{2}-b_{n(r-1)}\right)\left\|x_{n}-q\right\|^{2}+a_{n(r-1)}^{1} k_{n}^{2}\left[k_{n}^{2}\left\|x_{n}-q\right\|^{2}+\mu_{n}^{1}\right. \\
& \left.-a_{n r(1-}^{1}\left(1-a_{n r}^{1}-b_{n r}\right) g_{1}\left(\left\|T_{r}\left(P T_{r}\right)^{n-1} x_{n}-x_{n}\right\|\right)\right]+a_{n(r-1)}^{2} k_{n}^{2}\left\|x_{n}-q\right\|^{2} \\
& +b_{n(r-1)} M^{2} \\
& -a_{n(r-1)}^{1}\left(1-a_{n(r-1)}^{1}-a_{n(r-1)}^{2}-b_{n(r-1)}\right) g_{2}\left(\left\|T_{r-1}\left(P T_{r-1}\right)^{n-1} y_{n}-x_{n}\right\|\right) \\
\leq & k_{n}^{4}\left\|x_{n}-q\right\|^{2}+k_{n}^{2} \mu_{n}^{1}+b_{n(r-1)} M^{2} \\
& -a_{n(r-1)}^{1} a_{n r}^{1}\left(1-a_{n r}^{1}-b_{n r}\right) g_{1}\left(\left\|T_{r}\left(P T_{r}\right)^{n-1} x_{n}-x_{n}\right\|\right) \\
& -a_{n(r-1)}^{1}\left(1-a_{n(r-1)}^{1}-a_{n(r-1)}^{2}-b_{n(r-1)}\right) g_{2}\left(\left\|T_{r-1}\left(P T_{r-1}\right)^{n-1} y_{n}-x_{n}\right\|\right) \\
\leq & k_{n}^{4}\left\|x_{n}-q\right\|^{2}+\mu_{n}^{2} \\
& -a_{n(r-1)}^{1} a_{n r}^{1}\left(1-a_{n r}^{1}-b_{n r}\right) g_{1}\left(\left\|T_{r}\left(P T_{r}\right)^{n-1} x_{n}-x_{n}\right\|\right) \\
& -a_{n(r-1)}^{1}\left(1-a_{n(r-1)}^{1}-a_{n(r-1)}^{2}-b_{n(r-1)}\right) g_{2}\left(\left\|T_{r-1}\left(P T_{r-1}\right)^{n-1} y_{n}-x_{n}\right\|\right) \quad(2.7) \\
&
\end{aligned}
$$

where $\mu_{n}^{2}=k_{n}^{2} \mu_{n}^{1}+b_{n(r-1)} M^{2}$, so that $\sum_{n=1}^{\infty} \mu_{n}^{2}<\infty$. Proceeding in this way we have

$$
\begin{aligned}
\left\|y_{n+j}-q\right\|^{2} \leq & k_{n}^{2(j+1)}\left\|x_{n}-q\right\|^{2}+\mu_{n}^{(j+1)}- \\
& \left(\prod_{i=r-j}^{r} a_{n i}^{1}\right)\left(1-a_{n r}^{1}-b_{n r}\right) g_{1}\left(\left\|T_{r}\left(P T_{r}\right)^{n-1} x_{n}-x_{n}\right\|\right)- \\
& \left(\prod_{i=r-j}^{r-1} a_{n i}^{1}\right)\left(1-a_{n(r-1)}^{1}-a_{n(r-1)}^{2}-b_{n(r-1)}\right) g_{2}\left(\left\|T_{r-1}\left(P T_{r-1}\right)^{n-1} y_{n}-x_{n}\right\|\right)- \\
& \ldots .-a_{n(r-j)}^{1}\left(1-\sum_{k=1}^{j+1} a_{n(r-j)}^{k}-b_{n(r-j)}\right) g_{j+1}\left(\left\|T_{r-j}\left(P T_{r-j}\right)^{n-1} y_{n+j-1}-x_{n}\right\|\right)
\end{aligned}
$$

for $j=1,2, \ldots, r-2$ and $\left\{\mu_{n}^{(j+1)}\right\}$ is a nonnegative real sequence such that $\sum_{n=1}^{\infty} \mu_{n}^{(j+1)}<\infty$. Thus 
we get

$$
\begin{aligned}
\left\|x_{n+1}-q\right\|^{2} \leq & k_{n}^{2 r}\left\|x_{n}-q\right\|^{2}+\mu_{n}^{r}- \\
& \left(\prod_{i=1}^{r} a_{n i}^{1}\right)\left(1-a_{n r}^{1}-b_{n r}\right) g_{1}\left(\left\|T_{r}\left(P T_{r}\right)^{n-1} x_{n}-x_{n}\right\|\right)- \\
& \left(\prod_{i=1}^{r-1} a_{n i}^{1}\right)\left(1-a_{n(r-1)}^{1}-a_{n(r-1)}^{2}-b_{n(r-1)}\right) g_{2}\left(\left\|T_{r-1}\left(P T_{r-1}\right)^{n-1} y_{n}-x_{n}\right\|\right) \\
& -\ldots .-a_{n 1}^{1} a_{n 2}^{1}\left(1-\sum_{k=1}^{r-1} a_{n 2}^{k}-b_{n 2}\right) g_{r-1}\left(\left\|T_{2}\left(P T_{2}\right)^{n-1} y_{n+r-3}-x_{n}\right\|\right) \\
& -a_{n 1}^{1}\left(1-\sum_{k=1}^{r} a_{n 1}^{k}-b_{n 1}\right) g_{r}\left(\left\|T_{1}\left(P T_{1}\right)^{n-1} y_{n+r-2}-x_{n}\right\|\right)
\end{aligned}
$$

where $\left\{\mu_{n}^{r}\right\}$ is a nonnegative real sequence such that $\sum_{n=1}^{\infty} \mu_{n}^{r}<\infty$. Since $\left\{k_{n}\right\}$ is bounded so there exists $M_{1}>0$ such that $k_{n} \in\left[1, M_{1}\right]$ for all $n \geq 1$. Hence $k_{n}^{2 r}-1 \leq 2 r k_{n}^{2 r-1}\left(k_{n}-1\right) \leq$ $2 r M_{1}^{2 r-1}\left(k_{n}-1\right)$ holds for all $n \geq 1$. So $\sum_{n=1}^{\infty}\left(k_{n}-1\right)<\infty$ implies that $\sum_{n=1}^{\infty}\left(k_{n}^{2 r}-1\right)<\infty$. Therefore from (2.9) we get

$$
\begin{aligned}
\left\|x_{n+1}-q\right\|^{2} \leq & \left\|x_{n}-q\right\|^{2}+\left(k_{n}^{2 r}-1\right)\left\|x_{n}-q\right\|^{2}+\mu_{n}^{r}- \\
& \left(\prod_{i=1}^{r} a_{n i}^{1}\right)\left(1-a_{n r}^{1}-b_{n r}\right) g_{1}\left(\left\|T_{r}\left(P T_{r}\right)^{n-1} x_{n}-x_{n}\right\|\right)- \\
& \left(\prod_{i=1}^{r-1} a_{n i}^{1}\right)\left(1-a_{n(r-1)}^{1}-a_{n(r-1)}^{2}-b_{n(r-1)}\right) g_{2}\left(\left\|T_{r-1}\left(P T_{r-1}\right)^{n-1} y_{n}-x_{n}\right\|\right) \\
& -\ldots . a_{n 1}^{1} a_{n 2}^{1}\left(1-\sum_{k=1}^{r-1} a_{n 2}^{k}-b_{n 2}\right) g_{r-1}\left(\left\|T_{2}\left(P T_{2}\right)^{n-1} y_{n+r-3}-x_{n}\right\|\right) \\
& -a_{n 1}^{1}\left(1-\sum_{k=1}^{r} a_{n 1}^{k}-b_{n 1}\right) g_{r}\left(\left\|T_{1}\left(P T_{1}\right)^{n-1} y_{n+r-2}-x_{n}\right\|\right) \\
\leq & \left\|x_{n}-q\right\|^{2}+2 r M_{1}^{2 r-1}\left(k_{n}-1\right) D^{2}+\mu_{n}^{r}- \\
& \left(\prod_{i=1}^{r} a_{n i}^{1}\right)\left(1-a_{n r}^{1}-b_{n r}\right) g_{1}\left(\left\|T_{r}\left(P T_{r}\right)^{n-1} x_{n}-x_{n}\right\|\right)- \\
& \left(\prod_{i=1}^{r-1} a_{n i}^{1}\right)\left(1-a_{n(r-1)}^{1}-a_{n(r-1)}^{2}-b_{n(r-1)}\right) g_{2}\left(\left\|T_{r-1}\left(P T_{r-1}\right)^{n-1} y_{n}-x_{n}\right\|\right) \\
& -\ldots .-a_{n 1}^{1} a_{n 2}^{1}\left(1-\sum_{k=1}^{r-1} a_{n 2}^{k}-b_{n 2}\right) g_{r-1}\left(\left\|T_{2}\left(P T_{2}\right)^{n-1} y_{n+r-3}-x_{n}\right\|\right) \\
& \left.-\sum_{k=1}^{r} a_{n 1}^{k}-b_{n 1}\right) g_{r}\left(\left\|T_{1}\left(P T_{1}\right)^{n-1} y_{n+r-2}-x_{n}\right\|\right) . \\
& \\
& \\
& \\
&
\end{aligned}
$$


From (2.10) we get

$$
\begin{aligned}
& \left(\prod_{i=1}^{r} a_{n i}^{1}\right)\left(1-a_{n r}^{1}-b_{n r}\right) g_{1}\left(\left\|T_{r}\left(P T_{r}\right)^{n-1} x_{n}-x_{n}\right\|\right) \\
\leq & \left\|x_{n}-q\right\|^{2}-\left\|x_{n+1}-q\right\|^{2}+2 r M_{1}^{2 r-1}\left(k_{n}-1\right) D^{2}+\mu_{n}^{r}
\end{aligned}
$$

and

$$
\begin{aligned}
& \left(\prod_{i=1}^{j} a_{n i}^{1}\right)\left(1-\sum_{k=1}^{r-j+1} a_{n j}^{k}-b_{n j}\right) g_{r-j+1}\left(\left\|T_{j}\left(P T_{j}\right)^{n-1} y_{n+r-j-1}-x_{n}\right\|\right) \\
\leq & \left\|x_{n}-q\right\|^{2}-\left\|x_{n+1}-q\right\|^{2}+2 r M_{1}^{2 r-1}\left(k_{n}-1\right) D^{2}+\mu_{n}^{r}
\end{aligned}
$$

for $j=1,2, \ldots, r-1$. If $\liminf _{n \rightarrow \infty} a_{n i}^{1}>0$, for all $i<r$ and $0<\liminf _{n \rightarrow \infty} a_{n r}^{1} \leq \limsup _{n \rightarrow \infty}\left(a_{n r}^{1}+\right.$ $\left.b_{n r}\right)$, then there exists a positive integer $n_{0}$ and $\eta, \eta^{\prime} \in(0,1)$ such that $0<\eta<a_{n i}^{1}(i \in$ I), $a_{n r}^{1}+b_{n r}<\eta^{\prime}<1$, for all $n \geq n_{0}$. Thus from (2.11) we get

$$
\begin{aligned}
\eta^{r}\left(1-\eta^{\prime}\right) g_{1}\left(\left\|T_{r}\left(P T_{r}\right)^{n-1} x_{n}-x_{n}\right\|\right) \leq & \left\|x_{n}-q\right\|^{2}-\left\|x_{n+1}-q\right\|^{2}+2 r M_{1}^{2 r-1}\left(k_{n}-1\right) D^{2} \\
& +\mu_{n}^{r}, \text { for all } n \geq n_{0} .
\end{aligned}
$$

This implies that

$$
\begin{aligned}
\sum_{n=n_{0}}^{\infty} g_{1}\left(\left\|T_{r}\left(P T_{r}\right)^{n-1} x_{n}-x_{n}\right\|\right) \leq & \frac{1}{\eta^{r}\left(1-\eta^{\prime}\right)}\left(\left\|x_{n_{0}}-q\right\|^{2}+2 r M_{1}^{2 r-1} D^{2} \sum_{n=n_{0}}^{\infty}\left(k_{n}-1\right)\right. \\
& \left.+\sum_{n=n_{0}}^{\infty} \mu_{n}^{r}\right)<\infty
\end{aligned}
$$

which further implies that $\lim _{n \rightarrow \infty} g_{1}\left(\left\|T_{r}\left(P T_{r}\right)^{n-1} x_{n}-x_{n}\right\|\right)=0$. Since $g_{1}$ is strictly increasing and continuous with $g_{1}(0)=0$, so $\lim _{n \rightarrow \infty}\left\|T_{r}\left(P T_{r}\right)^{n-1} x_{n}-x_{n}\right\|=0$. Similarly from (2.12) using the fact that $g_{r-j+1}$ is strictly increasing and continuous with $g_{r-j+1}(0)=0$ we get $\lim _{n \rightarrow \infty}\left\|T_{j}\left(P T_{j}\right)^{n-1} y_{n+r-j-1}-x_{n}\right\|=0$ for $j=1,2, \ldots . ., r-1 . \diamond$

Lemma 2.3. Let $\mathrm{E}$ be a uniformly convex Banach space and $\mathrm{K}$ be a nonempty closed convex subset of $E$ which is also a nonexpansive retract of $E$. Let $T_{i}: K \rightarrow E(i \in I=\{1,2, \ldots, r\})$ be given nonself asymptotically nonexpansive mappings with sequences $\left\{k_{n}^{i}\right\} \subset[1, \infty)$ with $\sum_{n=1}^{\infty}\left(k_{n}^{i}-1\right)<\infty$ for $i \in$ I. Let $\left\{x_{n}\right\}$ be defined by (1.9) with $\sum_{n=1}^{\infty} b_{n i}<\infty$ for $i \in$ I. If $F=\bigcap_{i=1}^{r} F\left(T_{i}\right) \neq \emptyset$ and

(1) $0<\liminf _{n \rightarrow \infty} a_{n r}^{1} \leq \lim \sup _{n \rightarrow \infty}\left(a_{n r}^{1}+b_{n r}\right)<1$

(2) $0<\liminf _{n \rightarrow \infty} a_{n j}^{1} \leq \lim \sup _{n \rightarrow \infty}\left(\sum_{m=1}^{r-j+1} a_{n j}^{m}+b_{n j}\right)<1$,

then $\lim _{\mathfrak{n} \rightarrow \infty}\left\|x_{\mathfrak{n}}-T_{i} x_{n}\right\|=0$ for $i \in I$.

Proof: By Lemma 2.2 we get

$$
\begin{array}{r}
\lim _{n \rightarrow \infty}\left\|T_{r}\left(P T_{r}\right)^{n-1} x_{n}-x_{n}\right\|=0 \text { and } \\
\lim _{n \rightarrow \infty}\left\|T_{j}\left(P T_{j}\right)^{n-1} y_{n+r-j-1}-x_{n}\right\|=0 \text { for } j=1,2, \ldots ., r-1 .
\end{array}
$$


Since $\mathrm{P}$ is nonexpansive mapping so from (1.9) together with (2.13) we have

$$
\begin{aligned}
\left\|y_{n}-x_{n}\right\| & \leq a_{n r}^{1}\left\|T_{r}\left(P T_{r}\right)^{n-1} x_{n}-x_{n}\right\|+b_{n r}\left\|u_{n r}-x_{n}\right\| \\
& \leq\left\|T_{r}\left(P T_{r}\right)^{n-1} x_{n}-x_{n}\right\|+b_{n r}\left\|u_{n r}-x_{n}\right\| \rightarrow 0 \text { as } n \rightarrow \infty
\end{aligned}
$$

Since $T_{r-1}$ is nonself asymptotically nonexpansive mapping, from (2.13) and (2.14) we get

$$
\begin{aligned}
\left\|T_{r-1}\left(P_{r-1}\right)^{n-1} x_{n}-x_{n}\right\| \leq & \left\|T_{r-1}\left(P T_{r-1}\right)^{n-1} x_{n}-T_{r-1}\left(P T_{r-1}\right)^{n-1} y_{n}\right\|+ \\
& \left\|T_{r-1}\left(P T_{r-1}\right)^{n-1} y_{n}-x_{n}\right\| \\
\leq & k_{n}\left\|y_{n}-x_{n}\right\|+\left\|T_{r-1}\left(P T_{r-1}\right)^{n-1} y_{n}-x_{n}\right\| \\
\rightarrow & 0 \text { as } n \rightarrow \infty .
\end{aligned}
$$

Again from (1.9), 2.13) and (2.15) it follows that

$$
\begin{aligned}
\left\|y_{n+1}-x_{n}\right\| \leq & a_{n(r-1)}^{1}\left\|T_{r-1}\left(P T_{r-1}\right)^{n-1} y_{n}-x_{n}\right\|+a_{n(r-1)}^{2}\left\|T_{r-1}\left(P T_{r-1}\right)^{n-1} x_{n}-x_{n}\right\| \\
& +b_{n(r-1)}\left\|u_{n(r-1)}-x_{n}\right\| \rightarrow 0 \text { as } n \rightarrow \infty
\end{aligned}
$$

From (2.16) and (2.13) we have that

$$
\begin{aligned}
\left\|T_{r-2}\left(P T_{r-2}\right)^{n-1} x_{n}-x_{n}\right\| \leq & \left\|T_{r-2}\left(P T_{r-2}\right)^{n-1} x_{n}-T_{r-2}\left(P T_{r-2}\right)^{n-1} y_{n+1}\right\|+ \\
& \left\|T_{r-2}\left(P T_{r-2}\right)^{n-1} y_{n+1}-x_{n}\right\| \\
\leq & k_{n}\left\|y_{n+1}-x_{n}\right\|+\left\|T_{r-2}\left(P T_{r-2}\right)^{n-1} y_{n+1}-x_{n}\right\| \\
& \rightarrow 0 \text { as } n \rightarrow \infty .
\end{aligned}
$$

Also from (2.17) and (2.14) we have that

$$
\begin{aligned}
\left\|T_{r-2}\left(P T_{r-2}\right)^{n-1} y_{n}-x_{n}\right\| \leq & \left\|T_{r-2}\left(P T_{r-2}\right)^{n-1} y_{n}-T_{r-2}\left(P T_{r-2}\right)^{n-1} x_{n}\right\|+ \\
& \left\|T_{r-2}\left(P T_{r-2}\right)^{n-1} x_{n}-x_{n}\right\| \\
\leq & k_{n}\left\|y_{n}-x_{n}\right\|+\left\|T_{r-2}\left(P T_{r-2}\right)^{n-1} x_{n}-x_{n}\right\| \\
\rightarrow & 0 \text { as } n \rightarrow \infty .
\end{aligned}
$$

Continuing in this way we have that

$$
\lim _{n \rightarrow \infty}\left\|T_{j}\left(P T_{j}\right)^{n-1} y_{n+r-j-2}-x_{n}\right\|=0 \text { for } j=1,2, \ldots . ., r-2 .
$$

Again from (1.9), (2.13), (2.17) and (2.18) it follows that

$$
\begin{aligned}
\left\|y_{n+2}-x_{n}\right\| \leq & a_{n(r-2)}^{1}\left\|T_{r-2}\left(P T_{r-2}\right)^{n-1} y_{n+1}-x_{n}\right\|+a_{n(r-2)}^{2}\left\|T_{r-2}\left(P T_{r-2}\right)^{n-1} y_{n}-x_{n}\right\| \\
& +a_{n(r-2)}^{3}\left\|T_{r-2}\left(P T_{r-2}\right)^{n-1} x_{n}-x_{n}\right\|+b_{n(r-2)}\left\|u_{n(r-2)}-x_{n}\right\| \\
\rightarrow & 0 \text { as } n \rightarrow \infty
\end{aligned}
$$


From (2.20) and 2.13) we have that

$$
\begin{aligned}
\left\|T_{r-3}\left(P T_{r-3}\right)^{n-1} x_{n}-x_{n}\right\| \leq & \left\|T_{r-3}\left(P T_{r-3}\right)^{n-1} x_{n}-T_{r-3}\left(P T_{r-3}\right)^{n-1} y_{n+2}\right\|+ \\
& \left\|T_{r-3}\left(P T_{r-3}\right)^{n-1} y_{n+2}-x_{n}\right\| \\
\leq & k_{n}\left\|y_{n+2}-x_{n}\right\|+\left\|T_{r-3}\left(P T_{r-3}\right)^{n-1} y_{n+2}-x_{n}\right\| \\
\rightarrow & 0 \text { as } n \rightarrow \infty .
\end{aligned}
$$

Thus from (2.21) and (2.14) it follows that

$$
\begin{aligned}
\left\|T_{r-3}\left(P T_{r-3}\right)^{n-1} y_{n}-x_{n}\right\| \leq & \left\|T_{r-3}\left(P T_{r-3}\right)^{n-1} y_{n}-T_{r-3}\left(P T_{r-3}\right)^{n-1} x_{n}\right\|+ \\
& \left\|T_{r-3}\left(P T_{r-3}\right)^{n-1} x_{n}-x_{n}\right\| \\
\leq & k_{n}\left\|y_{n}-x_{n}\right\|+\left\|T_{r-3}\left(P T_{r-3}\right)^{n-1} x_{n}-x_{n}\right\| \\
\rightarrow & 0 \text { as } n \rightarrow \infty
\end{aligned}
$$

Continuing in this way we have that

$$
\lim _{n \rightarrow \infty}\left\|T_{j}\left(P T_{j}\right)^{n-1} y_{n+r-j-3}-x_{n}\right\|=0 \text { for } j=1,2, \ldots . ., r-3 .
$$

Continuing in this way after a finite steps we have that

$$
\begin{array}{r}
\lim _{n \rightarrow \infty}\left\|T_{i}\left(P T_{i}\right)^{n-1} x_{n}-x_{n}\right\|=0, \text { for } i \in I \text {, and } \\
\lim _{n \rightarrow \infty}\left\|T_{j}\left(P T_{j}\right)^{n-1} y_{n+r-j-k}-x_{n}\right\|=0 \text { for } j=1,2, \ldots ., r-k .
\end{array}
$$

From (1.9), 2.22) we have that

$$
\begin{aligned}
\left\|x_{n+1}-x_{n}\right\|= & \| P\left[\left(1-\sum_{k=1}^{r} a_{n 1}^{k}-b_{n 1}\right) x_{n}+\sum_{k=1}^{r-1} a_{n 1}^{k} T_{1}\left(P T_{1}\right)^{n-1} y_{n+r-1-k}+\right. \\
& \left.a_{n 1}^{r} T_{1}\left(P T_{1}\right)^{n-1} x_{n}+b_{n 1} u_{n 1}\right]-x_{n} \| \\
\leq & \sum_{k=1}^{r-1} a_{n 1}^{k}\left\|T_{1}\left(P T_{1}\right)^{n-1} y_{n+r-1-k}-x_{n}\right\|+a_{n 1}^{r}\left\|T_{1}\left(P T_{1}\right)^{n-1} x_{n}-x_{n}\right\| \\
& +b_{n 1}\left\|u_{n 1}-x_{n}\right\| \rightarrow 0 \text { as } n \rightarrow \infty .
\end{aligned}
$$

Since every nonself asymptotically nonexpansive mapping uniformly L-Lipschitzian, so from (2.22) and (2.23) we get

$$
\begin{aligned}
\left\|T_{i}\left(P T_{i}\right)^{n-2} x_{n}-x_{n}\right\| \leq & \left\|T_{i}\left(P T_{i}\right)^{n-2} x_{n}-T_{i}\left(P T_{i}\right)^{n-2} x_{n-1}\right\|+ \\
& \left\|T_{i}\left(P T_{i}\right)^{n-2} x_{n-1}-x_{n-1}\right\|+\left\|x_{n-1}-x_{n}\right\| \\
\leq & (1+L)\left\|x_{n}-x_{n-1}\right\|+\left\|T_{i}\left(P T_{i}\right)^{n-2} x_{n-1}-x_{n-1}\right\| \\
& \rightarrow 0 \text { as } n \rightarrow \infty .
\end{aligned}
$$

Now from (2.22) and 2.24) it follows that

$$
\begin{aligned}
\left\|x_{n}-T_{i} x_{n}\right\| & \leq\left\|x_{n}-T_{i}\left(P T_{i}\right)^{n-1} x_{n}\right\|+\left\|T_{i}\left(P T_{i}\right)^{n-1} x_{n}-T_{i} x_{n}\right\| \\
& \leq\left\|x_{n}-T_{i}\left(P T_{i}\right)^{n-1} x_{n}\right\|+L\left\|T_{i}\left(P T_{i}\right)^{n-2} x_{n}-x_{n}\right\| \\
& \rightarrow 0 \text { as } n \rightarrow \infty
\end{aligned}
$$


Thus we have that $\lim _{n \rightarrow \infty}\left\|x_{n}-T_{i} x_{n}\right\|=0$ for $i \in I . \diamond$

Lemma 2.4. Let $\mathrm{E}$ be a uniformly convex Banach space and $\mathrm{K}$ be a nonempty closed convex subset of $E$ which is also a nonexpansive retract of $E$ which has a Frèchet differentiable norm. Let $\mathrm{T}_{i}: \mathrm{K} \rightarrow \mathrm{E}(\mathrm{i} \in \mathrm{I}=\{1,2, \ldots, \mathrm{r}\})$ be given nonself asymptotically nonexpansive mappings with sequences $\left\{k_{n}^{i}\right\} \subset[1, \infty)$ with $\sum_{n=1}^{\infty}\left(k_{n}^{i}-1\right)<\infty$ for $i \in$ I. Let $\left\{x_{n}\right\}$ be defined by (1.9) with $\sum_{n=1}^{\infty} b_{n i}<\infty$ for $i \in$ I. If $F=\bigcap_{i=1}^{r} F\left(T_{i}\right) \neq \emptyset$ and

(1) $0<\liminf _{n \rightarrow \infty} a_{n r}^{1} \leq \limsup _{n \rightarrow \infty}\left(a_{n r}^{1}+b_{n r}\right)<1$

(2) $0<\liminf _{n \rightarrow \infty} a_{n j}^{1} \leq \lim \sup _{n \rightarrow \infty}\left(\sum_{m=1}^{r-j+1} a_{n j}^{m}+b_{n j}\right)<1$,

then for any $p_{1}, p_{2} \in F, \lim _{n \rightarrow \infty}<x_{n}, J\left(p_{1}-p_{2}\right)>$ exists. In particular $\lim _{n \rightarrow \infty}<p-q, J\left(p_{1}-p_{2}\right)>=0$ for all $p, q \in w_{w}\left(x_{n}\right)$.

Proof: Since $E$ has Frèchet differentiable norm, taking $x=p_{1}-p_{2}$ with $p_{1} \neq p_{2}$ and $h=t\left(x_{n}-p_{1}\right)$ in the inequality (1.11) we get that

$$
\begin{aligned}
& t<x_{n}-p_{1}, J\left(p_{1}-p_{2}\right)>+\frac{1}{2}\left\|p_{1}-p_{2}\right\|^{2} \leq \frac{1}{2}\left\|t x_{n}+(1-t) p_{1}-p_{2}\right\|^{2} \leq \\
& t<x_{n}-p_{1}, J\left(p_{1}-p_{2}\right)>+\frac{1}{2}\left\|p_{1}-p_{2}\right\|^{2}+b\left(t\left\|x_{n}-p_{1}\right\|\right) .
\end{aligned}
$$

Again $p_{1} \in F$, so by Lemma 2.1 we have that $\lim _{n \rightarrow \infty}\left\|x_{n}-p_{1}\right\|$ exists. Let $\sup \left\{\left\|x_{n}-p_{1}\right\|: n \in\right.$ $N\} \leq M^{\prime}$ for some $M^{\prime}>0$. Thus from [2.25) we get

$$
\begin{aligned}
& \frac{1}{2}\left\|p_{1}-p_{2}\right\|^{2}+\limsup _{n \rightarrow \infty} t<x_{n}-p_{1}, J\left(p_{1}-p_{2}\right)>\leq \frac{1}{2} \lim _{n \rightarrow \infty}\left\|t x_{n}+(1-t) p_{1}-p_{2}\right\|^{2} \\
& \leq \frac{1}{2}\left\|p_{1}-p_{2}\right\|^{2}+b\left(t M^{\prime}\right)+\liminf _{n \rightarrow \infty} t<x_{n}-p_{1}, J\left(p_{1}-p_{2}\right)> \\
\Rightarrow & \limsup _{n \rightarrow \infty}<x_{n}-p_{1}, J\left(p_{1}-p_{2}\right)>\leq \liminf _{n \rightarrow \infty}<x_{n}-p_{1}, J\left(p_{1}-p_{2}\right)>+\frac{b\left(t M^{\prime}\right)}{t M^{\prime}} M^{\prime} \\
\Rightarrow & \lim _{n \rightarrow \infty}<x_{n}-p_{1}, J\left(p_{1}-p_{2}\right)>\text { exists as } t \rightarrow 0 .
\end{aligned}
$$

In particular $\lim _{n \rightarrow \infty}<p-q, J\left(p_{1}-p_{2}\right)>=0$ for all $p, q \in w_{w}\left(x_{n}\right)$.

Lemma 2.5. Let $\mathrm{E}$ be a uniformly convex Banach space and $\mathrm{K}$ be a nonempty closed convex subset of $E$ which is also a nonexpansive retract of $E$. Let $T_{i}: K \rightarrow E(i \in I=\{1,2, \ldots, r\})$ be given nonself asymptotically nonexpansive mappings with sequences $\left\{k_{n}^{i}\right\} \subset[1, \infty)$ with $\sum_{n=1}^{\infty}\left(k_{n}^{i}-1\right)<\infty$ for $i \in$ I. Let $\left\{x_{n}\right\}$ be defined by (1.9) with $\sum_{n=1}^{\infty} b_{n i}<\infty$ for $i \in$ I. If $F=\bigcap_{i=1}^{r} F\left(T_{i}\right) \neq \emptyset$ and

(1) $0<\liminf _{n \rightarrow \infty} a_{n r}^{1} \leq \lim \sup _{n \rightarrow \infty}\left(a_{n r}^{1}+b_{n r}\right)<1$

(2) $0<\liminf _{n \rightarrow \infty} a_{n j}^{1} \leq \lim \sup _{n \rightarrow \infty}\left(\sum_{m=1}^{r-j+1} a_{n j}^{m}+b_{n j}\right)<1$,

then $\lim _{n \rightarrow \infty}\left\|t x_{n}+(1-t) p_{1}-p_{2}\right\|$ exists for all $p_{1}, p_{2} \in F$. 
Proof: Let $d_{n}(t)=\left\|t x_{n}+(1-t) p_{1}-p_{2}\right\|$ for all $t \in[0,1]$ and $p_{1}, p_{2} \in F$. Then $\lim _{n \rightarrow \infty} d_{n}(0)=\left\|p_{1}-p_{2}\right\|$ exists and $\lim _{n \rightarrow \infty} d_{n}(1)=\left\|x_{n}-p_{2}\right\|$ exists by Lemma 2.1, Define $Q_{n}: K \rightarrow E$ by

$$
\begin{aligned}
\mathrm{Q}_{n} x= & P\left[\left(1-a_{n 1}^{1}-a_{n 1}^{2}-\ldots . .-a_{n 1}^{r}-b_{n 1}\right) x+a_{n 1}^{1} T_{1}\left(P T_{1}\right)^{n-1} x_{r-2}+\right. \\
& \left.a_{n 1}^{2} T_{1}\left(P T_{1}\right)^{n-1} x_{r-3}+\ldots . .+a_{n 1}^{r} T_{1}\left(P T_{1}\right)^{n-1} x+b_{n 1} u_{n 1}\right] \\
x_{r-2}= & P\left[\left(1-a_{n 2}^{1}-a_{n 2}^{2}-\ldots . .-a_{n 2}^{r-1}-b_{n 2}\right) x+a_{n 2}^{1} T_{2}\left(P T_{2}\right)^{n-1} x_{r-3}+\right. \\
& \left.a_{n 2}^{2} T_{2}\left(P T_{2}\right)^{n-1} x_{r-4}+\ldots . .+a_{n 2}^{r-1} T_{2}\left(P T_{2}\right)^{n-1} x+b_{n 2} u_{n 2}\right] \\
. & \\
\cdot & \\
\cdot & \\
x_{2}= & P\left[\left(1-a_{n(r-2)}^{1}-a_{n(r-2)}^{2}-a_{n(r-2)}^{3}-b_{n(r-2)}\right) x+a_{n(r-2)}^{1} T_{r-2}\left(P T_{r-2}\right)^{n-1} x_{1}\right. \\
& \left.+a_{n(r-2)}^{2} T_{r-2}\left(P T_{r-2}\right)^{n-1} x_{0}+a_{n(r-2)}^{3} T_{r-2}\left(P T_{r-2}\right)^{n-1} x+b_{n(r-2)} u_{n(r-2)}\right] \\
x_{1}= & P\left[\left(1-a_{n(r-1)}^{1}-a_{n(r-1)}^{2}-b_{n(r-1)}\right) x+a_{n(r-1)}^{1} T_{r-1}\left(P T_{r-1}\right)^{n-1} x_{0}+\right. \\
& \left.a_{n(r-1)}^{2} T_{r-1}\left(P T_{r-1}\right)^{n-1} x+b_{n(r-1)} u_{n(r-1)}\right] \\
x_{0}= & P\left[\left(1-a_{n r}^{1}-b_{n r}\right) x+a_{n r}^{1} T_{r}\left(P T_{r}\right)^{n-1} x+b_{n r} u_{n r}\right]
\end{aligned}
$$

for all $x \in \mathrm{K}$. Thus for all $x, z \in \mathrm{K}$

$$
\begin{aligned}
\left\|x_{0}-z_{0}\right\| & \leq\left(1-a_{n r}^{1}-b_{n r}\right)\|x-z\|+a_{n r}^{1}\left\|T_{r}\left(P T_{r}\right)^{n-1} x-T_{r}\left(P_{r}\right)^{n-1} z\right\| \\
& \leq\left(1-a_{n r}^{1}-b_{n r}\right)\|x-z\|+a_{n r}^{1} k_{n}\|x-z\| \\
& \leq k_{n}\|x-z\| .
\end{aligned}
$$

Proceeding in this way we get

$$
\left\|\mathrm{Q}_{\mathrm{n}} x-\mathrm{Q}_{\mathrm{n}} z\right\| \leq \mathrm{k}_{\mathrm{n}}^{\mathrm{r}}\|x-z\|=\left[1+\left(\mathrm{k}_{\mathrm{n}}^{\mathrm{r}}-1\right)\right]\|x-z\| .
$$

Set

$$
\begin{aligned}
& S_{n, m}=Q_{n+m-1} Q_{n+m-2} \ldots \ldots . Q_{n}, m \geq 1 \text { and } \\
& b_{n, m}=\left\|S_{n, m}\left(t x_{n}+(1-t) p_{1}\right)-\left(t x_{n+m}+(1-t) p_{1}\right)\right\| .
\end{aligned}
$$

Then

$$
\left\|S_{n, m} x-S_{n, m} y\right\| \leq\left(\prod_{j=n}^{n+m-1} k_{j}^{r}\right)\|x-y\|=H_{n m r}\|x-y\|
$$

where $H_{n m r}=\left(\prod_{j=n}^{n+m-1} k_{j}^{r}\right)$ for $n \geq 1, S_{n, m} x_{n}=x_{n+m}$ and $S_{n, m} p=p$ for all $p \in F$. From the facts $\sum_{n=1}^{\infty}\left(k_{n}-1\right)<\infty$ and $0 \leq t^{r}-1 \leq r t^{r-1}(t-1)$ for all $t \geq 1$ we have that $\sum_{n=1}^{\infty}\left(k_{n}^{r}-1\right)<\infty$ 
which in turn implies that $H_{n m r} \rightarrow 1$ as $n, m \rightarrow \infty$. Also we have that $S_{n, m}$ is Lipschitzian with the lipschitz constant $\mathrm{H}_{\mathrm{nmr}}$. By Lemma 1.7 we have

$$
\begin{aligned}
b_{n, m} & \leq H_{n m r} \phi^{-1}\left(\left\|x_{n}-p_{1}\right\|-H_{n m r}^{-1}\left\|S_{n, m} x_{n}-S_{n, m} p_{1}\right\|\right) \\
& =H_{n m r} \phi^{-1}\left(\left\|x_{n}-p_{1}\right\|-H_{n m r}^{-1}\left\|x_{n+m}-p_{1}\right\|\right) .
\end{aligned}
$$

Now,

$$
\begin{aligned}
d_{n+m}(t) & =\left\|t x_{n+m}+(1-t) p_{1}-p_{2}\right\| \\
& \leq b_{n, m}+\left\|S_{n, m}\left(t x_{n}+(1-t) p_{1}\right)-p_{2}\right\| \\
& =b_{n, m}+\left\|S_{n, m}\left(t x_{n}+(1-t) p_{1}\right)-S_{n, m} p_{2}\right\| \\
& \leq b_{n, m}+H_{n m r}\left\|t x_{n}+(1-t) p_{1}-p_{2}\right\| \\
& =b_{n, m}+H_{n m r} d_{n}(t)
\end{aligned}
$$

It then follows from Lemma 2.1 that the sequence $\left\{b_{n, m}\right\}$ converges uniformly to 0 as $n \rightarrow \infty$ for all $m \geq 1$. Thus from above we get

$$
\limsup _{n \rightarrow \infty} d_{n}(t) \leq \phi^{-1}(0)+\liminf _{n \rightarrow \infty} d_{n}(t)=\liminf _{n \rightarrow \infty} d_{n}(t) .
$$

This completes the proof.

Theorem 1. Let $\mathrm{E}$ be a uniformly convex Banach space and $\mathrm{K}$ be a nonempty closed convex subset of $E$ which is also a nonexpansive retract of $E$. Let $T_{i}: K \rightarrow E(i \in I=\{1,2, \ldots, r\})$ be given nonself asymptotically nonexpansive mappings with sequences $\left\{k_{n}^{i}\right\} \subset[1, \infty)$ with $\sum_{n=1}^{\infty}\left(k_{n}^{i}-1\right)<\infty$ for $i \in$ I. Let $\left\{x_{n}\right\}$ be defined by (1.9) with $\sum_{n=1}^{\infty} b_{n i}<\infty$ for $i \in I$. Let $F=\bigcap_{i=1}^{r} F\left(T_{i}\right) \neq \emptyset$ and

(1) $0<\liminf _{n \rightarrow \infty} a_{n r}^{1} \leq \limsup \sup _{n \rightarrow \infty}\left(a_{n r}^{1}+b_{n r}\right)<1$

(2) $0<\liminf _{n \rightarrow \infty} a_{n j}^{1} \leq \lim \sup _{n \rightarrow \infty}\left(\sum_{m=1}^{r-j+1} a_{n j}^{m}+b_{n j}\right)<1$.

Assume that any one of the following conditions holds:

(1)E satisfies Opial's property

(2)E has a Frechet differentiable norm

(3) $E^{\star}$ has the Kadec-Klee property

then $\left\{x_{n}\right\}$ converges weakly to some common fixed point of $\left\{T_{i}\right\}, i \in I$.

Proof: Since $F \neq \emptyset$, so let $q \in F$. Then by Lemma $2.1 \lim _{n \rightarrow \infty}\left\|x_{n}-q\right\|$ exists and so $\left\{x_{n}\right\}$ is bounded. Since $E$ be a uniformly convex Banach space so $\left\{x_{n}\right\}$ has a subsequence $\left\{x_{n_{j}}\right\}$ which is weakly convergent to $p \in K$ (say). From Lemma 2.3 we get $\lim _{n \rightarrow \infty}\left\|x_{n}-T_{i} x_{n}\right\|=0$ for $i \in I$. By Lemma 1.5 we have $T_{i}$ is demiclosed at 0 so $p \in F\left(T_{i}\right)$ for all $i \in I$. Then $p \in F$. If possible let $\left\{x_{n}\right\}$ has another subsequence $\left\{x_{n_{k}}\right\}$ which converges weakly to another point $q \in K$. Then by similar 
argument as above we have that $q \in F(T)$. Let (1) hold. Then by Opial's property we have

$$
\begin{aligned}
\left\|x_{n}-p\right\| & =\limsup _{j \rightarrow \infty}\left\|x_{n_{j}}-p\right\| \\
& <\limsup _{j \rightarrow \infty}\left\|x_{n_{j}}-q\right\|=\lim _{n \rightarrow \infty}\left\|x_{n}-q\right\| \\
& =\limsup _{k \rightarrow \infty}\left\|x_{n_{k}}-q\right\| \\
& <\limsup _{k \rightarrow \infty}\left\|x_{n_{k}}-p\right\|=\lim _{n \rightarrow \infty}\left\|x_{n}-p\right\|
\end{aligned}
$$

a contradiction. So $p=q$.

Let (2) hold. Then from Lemma 2.4 we get $\lim _{n \rightarrow \infty}<p-q, J\left(p_{1}-p_{2}\right)>=0$ for all $p, q \in$ $\mathrm{w}_{w}\left(x_{n}\right)$ and $p_{1}, p_{2} \in F$. Since $p, q \in F$ also so from above we get $<p-q, J(p-q)>=0$, that is, $\|\mathrm{p}-\mathrm{q}\|^{2}=0$ which implies that $\mathrm{p}=\mathrm{q}$.

Let (3) hold. Then from Lemma 2.5 we get $\lim _{n \rightarrow \infty}\left\|t x_{n}+(1-t) p-q\right\|$ exists, so by Lemma 1.6 we have that $p=q$. So $\left\{x_{n}\right\}$ converges weakly to some common fixed point of $\left\{T_{i}\right\}, i \in I$. This completes the proof of the Theorem. $\diamond$

Condition $(A)$ [14 A mapping $T: K \rightarrow E$ with nonempty fixed point set $F(T)$ in $K$ satisfies Condition $(A)$ if there is a nondecreasing function $f:[0, \infty) \rightarrow[0, \infty)$ with $f(0)=0$ and $f(m)>0$ for all $m \in(0, \infty)$ such that

$$
f(d(x, F(T))) \leq\|x-T x\| \text { for all } x \in K .
$$

A finite family of mappings $T_{i}: K \rightarrow E$, for all $i=1,2,3, \ldots, r$ with nonempty fixed point set $\mathrm{F}=\bigcap_{i=1}^{r} \mathrm{~F}\left(\mathrm{~T}_{\mathrm{i}}\right) \neq \emptyset$ satisfies

(i) Condition $(\bar{A})$ [4 if there is a nondecreasing function $f:[0, \infty) \rightarrow[0, \infty)$ with $f(0)=0$ and $f(m)>0$ for all $m \in(0, \infty)$ such that

$$
f(d(x, F)) \leq \frac{1}{r}\left(\sum_{i=1}^{r}\left\|x-T_{i} x\right\|\right) \text { for all } x \in K
$$

(ii) Condition $(\bar{B})[\underline{4}$ if there is a nondecreasing function $f:[0, \infty) \rightarrow[0, \infty)$ with $f(0)=0$ and $f(m)>0$ for all $m \in(0, \infty)$ such that

$$
f(d(x, F)) \leq \max _{1 \leq i \leq r}\left\{\left\|x-T_{i} x\right\|\right\} \text { for all } x \in K
$$

(iii) Condition $(\overline{\mathrm{C}})[4$ if there is a nondecreasing function $f:[0, \infty) \rightarrow[0, \infty)$ with $f(0)=0$ and $f(m)>0$ for all $m \in(0, \infty)$ such that at least one of the $T_{i}$ 's satisfies condition $(A)$.

Clearly if $T_{i}=T$, for all $i=1,2,3, \ldots, r$, then Condition $(\bar{A})$ reduces to Condition $(A)$. Also Condition $(\bar{B})$ reduces to Condition $(A)$ if all but one of $T_{i}$ 's are identities. Also it contains Condition $(\bar{A})$. Furthermore Condition $(\overline{\mathrm{C}})$ and Condition $(\overline{\mathrm{B}})$ are equivalent. Tan and $\mathrm{Xu}$ [18] pointed out that the Condition $(A)$ is weaker than the compactness of $K$. It is well known that every continuous and demicompact mapping must satisfy Condition(A) [14]. Since every completely continuous mapping is continuous and demicompact so it must satisfy Condition $(A)$. Also Condition $(\bar{B})$ contains 
Condition $(\bar{A})$ therefore to study the strong convergence of the iterative sequence $\left\{x_{n}\right\}$ be defined by (1.9) we use Condition $(\bar{B})$ instead of the complete continuity of the mappings $\left\{T_{1}, T_{2}, \ldots ., T_{r}\right\}$ and Condition $(\overline{\mathcal{A}})$.

Theorem 2. Let $\mathrm{E}$ be a uniformly convex Banach space and $\mathrm{K}$ be a nonempty closed convex subset of $E$ which is also a nonexpansive retract of $E$. Let $T_{i}: K \rightarrow E(i \in I=\{1,2, \ldots, r\})$ be given nonself asymptotically nonexpansive mappings with sequences $\left\{k_{n}^{i}\right\} \subset[1, \infty)$ with $\sum_{n=1}^{\infty}\left(k_{n}^{i}-1\right)<\infty$ for $i \in$ I. Let $\left\{x_{n}\right\}$ be defined by [1.9] with $\sum_{n=1}^{\infty} b_{n i}<\infty$ for $i \in I$. Let $F=\bigcap_{i=1}^{r} F\left(T_{i}\right) \neq \emptyset$ and

(1) $0<\liminf _{n \rightarrow \infty} a_{n r}^{1} \leq \limsup _{n \rightarrow \infty}\left(a_{n r}^{1}+b_{n r}\right)<1$

(2) $0<\liminf _{n \rightarrow \infty} a_{n j}^{1} \leq \lim \sup _{n \rightarrow \infty}\left(\sum_{m=1}^{r-j+1} a_{n j}^{m}+b_{n j}\right)<1$.

If the family of mappings $\left\{T_{1}, T_{2}, \ldots ., T_{r}\right\}$ satisfy Condition $(\bar{B})$, then $\left\{x_{n}\right\}$ converges strongly to some common fixed point of $\left\{T_{1}, T_{2}, \ldots ., T_{r}\right\}$.

Proof: Let $\mathrm{q} \in \mathrm{F}$ then by Lemma $2.1 \lim _{n \rightarrow \infty}\left\|x_{n}-\mathrm{q}\right\|$ exists. Let $\lim _{n \rightarrow \infty}\left\|x_{n}-q\right\|=a$, for some $a \geq 0$. Let $a>0$. Now from (2.5) we get

$$
\begin{aligned}
\left\|x_{n+1}-q\right\| & \leq\left[1+\left(k_{n}^{r}-1\right)\right]\left\|x_{n}-q\right\|+\sigma_{n}^{r} \\
& =\left[1+\delta_{n}\right]\left\|x_{n}-q\right\|+\sigma_{n}^{r}
\end{aligned}
$$

where $\left\{\sigma_{n}^{r}\right\}$ is a nonnegative real sequence such that $\sum_{n=1}^{\infty} \sigma_{n}^{r}<\infty$ and $\delta_{n}=k_{n}^{r}-1$ such that $\sum_{n=1}^{\infty} \delta_{n}<\infty$. So

$$
d\left(x_{n+1}, F\right) \leq\left(1+\delta_{n}\right) d\left(x_{n}, F\right)+\sigma_{n}^{r} .
$$

By Lemma 1.1 we have that $\lim _{n \rightarrow \infty} d\left(x_{n}, F\right)$ exists. By Condition $(\bar{B})$ and Lemma 2.3 we get,

$$
\lim _{n \rightarrow \infty} f\left(d\left(x_{n}, F\right)\right)=0 .
$$

Since $f:[0, \infty) \rightarrow[0, \infty)$ is a nondecreasing function with $f(0)=0$ so we have

$\lim _{n \rightarrow \infty} d\left(x_{n}, F\right)=0$. Since $\lim _{n \rightarrow \infty}\left\|x_{n}-q\right\|$ exists, it follows that $\left\{\left\|x_{n}-q\right\|\right\}$ is bounded, so there exists $M^{\prime \prime}>0$ such that $\left\|x_{n}-q\right\| \leq M^{\prime \prime}$. From [2.26) we get

$$
\begin{aligned}
\left\|x_{n+1}-q\right\| & \leq\left\|x_{n}-q\right\|+\delta_{n} M^{\prime \prime}+\sigma_{n}^{r} \\
& =\left\|x_{n}-q\right\|+\theta_{n}
\end{aligned}
$$

where $\theta_{n}=\delta_{n} M^{\prime \prime}+\sigma_{n}^{r}$. Now $\sum_{n=1}^{\infty} \theta_{n}<\infty$. Now for any $m>1$ we have that

$$
\begin{aligned}
\left\|x_{n+m}-\mathrm{q}\right\| \leq & \left\|x_{n+m-1}-\mathrm{q}\right\|+\theta_{n+m-1} \\
\leq & \left\|x_{n+m-2}-\mathrm{q}\right\|+\theta_{n+m-2}+\theta_{n+m-1} \\
& \cdots \cdots \cdots \cdots \cdots \cdots \cdots \cdots \cdots \cdots \cdots \cdots \cdots \cdots \cdots \\
\leq & \left\|x_{n}-q\right\|+\sum_{k=n}^{n+m-1} \theta_{k} .
\end{aligned}
$$


Since $\sum_{n=1}^{\infty} \theta_{n}<\infty$ and $\lim _{n \rightarrow \infty} d\left(x_{n}, F\right)=0$, there exists $N_{1} \in N$ such that for all $n \geq N_{1}$ we have $d\left(x_{n}, F\right)<\frac{\epsilon}{3}$ and $\sum_{n=N_{1}}^{\infty} \theta_{n}<\frac{\epsilon}{6}$. Therefore there exists $\bar{x} \in F$ such that $\left\|x_{N_{1}}-\bar{x}\right\|<\frac{\epsilon}{3}$. Therefore we have

$$
\begin{aligned}
\left\|x_{n+m}-x_{n}\right\| & \leq\left\|x_{n+m}-\bar{x}\right\|+\left\|x_{n}-\bar{x}\right\| \\
& <\left\|x_{N_{1}}-\bar{x}\right\|+\sum_{k=N_{1}}^{n+m-1} \theta_{k}+\left\|x_{N_{1}}-\bar{x}\right\|+\sum_{k=N_{1}}^{n-1} \theta_{k} \\
& <\frac{\epsilon}{3}+\frac{\epsilon}{6}+\frac{\epsilon}{3}+\frac{\epsilon}{6}=\epsilon
\end{aligned}
$$

,.nonumber

Hence $\left\{x_{n}\right\}$ is a Cauchy sequence. Since $E$ is complete so $x_{n} \rightarrow p \in E$, so for given $\epsilon>0$ there exists $n_{1} \in N$ such that for all $n \geq n_{1},\left\|x_{n}-p\right\| \leq \frac{\epsilon}{2\left(1+k_{1}\right)}$. Again since $\lim _{n \rightarrow \infty} d\left(x_{n}, F\right)=0$, so for given $\epsilon>0$ there exists $n_{2} \in N$ such that for all $n \geq n_{2}\left(\geq n_{1}\right), d\left(x_{n}, F\right)<\frac{\epsilon}{2\left(1+k_{1}\right)}$. so there exists $\bar{p} \in \mathrm{F}$ such that $\left\|x_{n_{2}}-\bar{p}\right\| \leq \frac{\epsilon}{2\left(1+k_{1}\right)}$. Therefore

$$
\begin{aligned}
\left\|p-T_{i} p\right\| & =\left\|p-x_{n_{2}}+x_{n_{2}}-\bar{p}+\bar{p}-T_{i} p\right\| \\
& \leq\left\|p-x_{n_{2}}\right\|+\left\|x_{n_{2}}-\bar{p}\right\|+\left\|\bar{p}-T_{i} p\right\| \\
& =\left\|p-x_{n_{2}}\right\|+\left\|x_{n_{2}}-\bar{p}\right\|+\left\|T_{i} \bar{p}-T_{i} p\right\| \\
& \leq\left\|p-x_{n_{2}}\right\|+\left\|x_{n_{2}}-\bar{p}\right\|+k_{1}\|\bar{p}-p\| \\
& \leq\left(1+k_{1}\right)\left\|p-x_{n_{2}}\right\|+\left(1+k_{1}\right)\left\|x_{n_{2}}-\bar{p}\right\| \\
& \leq\left(1+k_{1}\right) \frac{\epsilon}{2\left(1+k_{1}\right)}+\left(1+k_{1}\right) \frac{\epsilon}{2\left(1+k_{1}\right)}=\epsilon .
\end{aligned}
$$

Since $\epsilon$ is arbitrary so we have $T_{i} p=p$ for all $i \in I$. So $p \in F\left(T_{i}\right)$ for all $i \in I$. Thus $p \in F$. Hence $\left\{x_{n}\right\}$ converges strongly to some common fixed point of $\left\{T_{1}, T_{2}, \ldots ., T_{r}\right\}$.

Remark 2.6. Theorem 1 and Theorem 2 extends and generalize Theorem 2.1 and Theorem 2.5 of [20.

\section{ACKNOWLEDGEMENT}

This work is supported by Council of Scientific and Industrial Research(CSIR), Government of India .

Received: December 2011. Revised: September 2012.

\section{References}

[1] R.E.Bruck, A simple proof of the mean ergodic theorem for nonlinear contractions in Banach spaces, Israel J.Math. 32(1979),107-116. 
[2] C.E.Chidume, E.U.Ofoedu, H.Zegeye, Strong and weak convergence theorems for asymptotically nonexpansive mappings, J. Math. Anal. Appl. 280(2003)364-374.

[3] C.E.Chidume, Bashir Ali, Approximation of common fixed points for finite families of nonself asymptotically nonexpansive mappings in Banach spaces, J. Math. Anal. Appl. 326(2007)960973.

[4] C.E.Chidume, Bashir Ali, Weak and strong convergence theorems for finite families of asymptotically nonexpansive mappings in Banach spaces, J. Math. Anal. Appl. 330(2007)377-387.

[5] Y.J.Cho, H.Y.Zhou, G.Guo; Weak and strong convergence theorems for three-step iterations with errors for asymptotically nonexpansive mappings, Comput. Math. Appl. 47(2004)707-717.

[6] J.G.Falset, W.Kaczor, T.Kuczumow, S.Reich, Weak convrgence theorems for asymptotically nonexpansive mappings and semigroups, Nonlinear Anal.43(2001)377-401.

[7] K.Goebel and W.A.Kirk; A fixed point theorem for asymptotically nonexpansive mappings, Proc. Amer. Math. Soc. 35(1972), 171-174.

[8] S.Ishikawa; Fixed points by a new iteration, Proc. Amer. Math. Soc. 44(1974), 147-150.

[9] J.U.Jeong, S.H.Kim, Weak and strong convergence of the Ishikawa iteration process with errors for two asymptotically nonexpansive mappings, Appl. Math. Comp. 181(2006)1394-1401.

[10] W.Kaczor, Weak convergence of almost orbits of asymptotically nonexpansive mappings, J.Math.Anal.Appl. 272(2002)565-574.

[11] W.R.Mann, Mean value methods in iteration, Proc. Amer. Math. Soc. 4(1953),506-510.

[12] Z.Opial, Weak convergence of the sequence of successive approximation for nonexpansive mappings, Bull. Amer. Math. Soc. 73(1967)591-597.

[13] M.O.Osilike, A.Udomene, Demiclosedness principle and convergence theorems for strictly pseudocontractive mappings of Browder-Petryshyn type, J. Math. Anal. Appl. 256(2001)431445 .

[14] H.F.Senter and W.G.Dotson, Jr., Approximating fixed points of nonexpansive mappings, Proc. Amer. Math. Soc. 44,375-380,(1974).

[15] N.Shahzad, Approximating fixed points of nonself nonexpansive mappings in Banach spaces, Nonlinear Anal.61(2005)1031-1039.

[16] S.Y.Matsushita, D.Kuroiwa, Strong convergence of averaging iteration of nonexpansive nonself-mappings, J. Math. Anal. Appl. 294(2004)206-214.

[17] W.Takahashi, G.E.Kim, Strong convergence of approximants to fixed points of nonexpansive nonself-mappings in Banach spaces, Nonlinear Anal.3(32)(1998)447-454. 
[18] K.K.Tan, H.K.Xu, Approximating fixed points of nonexpansive mapping by the Ishikawa iteration process, J. Math. Anal. Appl. 178(1993)301-308.

[19] L.Wang, Strong and weak convergence theorems for common fixed points of nonself asymptotically nonexpansive mappings, J. Math. Anal. Appl. 323(2006)550-557.

[20] L.Yang, Modified multistep iterative for some common fixed point of a finite family of nonself asymptotically nonexpansive mappings, Math. Comput. Modelling 45(2007)1157-1169.

[21] H.K.Xu, Inequalities in Banach spaces with applications, Nonlinear Anal. 16(1991) 1127-1138. 